\title{
Equivariant Surgery Theory for Homology Equivalences under the Gap Condition
}

\author{
Dedicated to Professor Yasuhiko Kitada on his 60th birthday
}

By

Masaharu MoRimoto*

\begin{abstract}
In the present paper, we discuss an obstruction theory to modify equivariant framed maps on even-dimensional compact smooth manifolds to homology equivalences by equivariant surgery. In 1974, Cappell-Shaneson already developed such obstruction theory in the nonequivariant setting. Our definition of the surgeryobstruction group presents a new aspect of Cappell-Shaneson's group in the nonequivariant setting and enables us to define directly the surgery obstructions of certain framed maps that are not necessarily connected up to the middle dimension. Using our framework defining the equivariant surgery obstruction, we prove a basic conjecture related to geometric connected sums and algebraic sums of surgery obstructions.
\end{abstract}

\section{$\S 1 . \quad$ Introduction}

Throughout the present paper, let $G$ be a finite group and $Y$ a compact, connected, oriented, smooth $G$-manifold of even dimension $n=2 k \geq 6$. We set

$$
\widetilde{G}_{Y}=\pi_{1}\left(E G \times_{G} Y\right),
$$

where $E G$ is a contractible $G$-CW complex with free $G$-action. Let $\zeta$ denote the canonical homomorphism $\widetilde{G}_{Y} \rightarrow G\left(G=\pi_{1}(B G)\right)$. The group $\widetilde{G}_{Y}$ acts

Communicated by K. Saito. Received January 24, 2005.

2000 Mathematics Subject Classification(s): Primary 57R67, 57S17; Secondary 19J25, $20 \mathrm{C} 05$.

Key words: Equivariant surgery, surgery obstruction, gap condition, homology equivalence.

Partially supported by a Grant-in-Aid for Scientific Research (KAKENHI).

* Graduate School of Natural Science and Technology \& Faculty of Environmental Science and Technology, Okayama University, 3-1-1 Tsushimanaka, Okayama 700-8530, Japan. 
on the universal covering space $\tilde{Y}$ of $Y$ so that the projection map $\widetilde{Y} \rightarrow Y$ is $\zeta$-equivariant (see [4]).

Each oriented $G$-manifold $Z$ determines the orientation homomorphism $w_{Z}: G \rightarrow\{1,-1\}$. The value $w_{Z}(g)$ at $g \in G$ is equal to 1 if and only if the translation $Z \rightarrow Z$ by $g$ is orientation preserving. Thus we have the orientation homomorphisms $w_{Y}: G \rightarrow\{1,-1\}$ and $w_{\widetilde{Y}}: \widetilde{G}_{Y} \rightarrow\{1,-1\}$. As usual, the orientation homomorphisms $w_{Y}, w_{\widetilde{Y}}$ and $w_{Z}$ etc. will be abbreviated to $w$ if what $w$ means is clear from the context. Let $R$ denote either $\mathbb{Z}$ or $\mathbb{Z}_{(p)}$ for a prime $p$, where

$$
\mathbb{Z}_{(p)}=\left\{\frac{a}{b} \in \mathbb{Q} \mid a \in \mathbb{Z}, b \in \mathbb{N},(p, b)=1\right\} .
$$

The orientation homomorphism $w_{Z}$ above induces an involution $\rho_{Z}$ on $R[G]$ such that $\rho_{Z}(r g)=w(g) r g^{-1}$ for $r \in R, g \in G$ and $\rho_{Z}(a+b)=\rho_{Z}(a)+\rho_{Z}(b)$ for $a, b \in R[G]$. In the following, the group rings $R[G]$ and $R\left[\widetilde{G}_{Y}\right]$ are equipped with the involutions $\rho_{Y}$ and $\rho_{\widetilde{Y}}$, respectively. As usual, - will be used for the involutions $\rho_{\tilde{Y}}, \rho_{Y}$ and $\rho_{Z}$ etc. if what - means is clear from the context.

We define the singular set $Y_{\text {sing }}$ and the regular set $Y_{\text {reg }}$ of $Y$ by

$$
Y_{\text {sing }}=\bigcup_{g \in G \backslash\{e\}} Y^{g} \text { and } Y_{\text {reg }}=Y \backslash\left(\partial Y \cup Y_{\text {sing }}\right) \text {, }
$$

respectively.

Let $f: X \rightarrow Y$ be a degree-one, one-connected, $G$-framed map satisfying the gap condition

$$
\operatorname{dim} X^{g}<\operatorname{dim} X / 2 \text { for all } g \in G \backslash\{e\},
$$

where $X$ is a compact, connected, oriented, smooth $G$-manifold of dimension $n$ and $f$ is covered by a $G$-vector bundle map $T(X) \oplus f^{*} \eta \rightarrow \xi$ for some $G$ vector bundles $\eta$ and $\xi$ over $Y$. Since $f$ is one-connected, the induced map $\pi_{1}(X) \rightarrow \pi_{1}(Y)$ must be surjective. We give the precise definition of a $G$ framed map in Section 2. Since $f: X \rightarrow Y$ is a degree-one map, the orientation homomorphism $G \rightarrow\{1,-1\}$ given by $X$ coincides with that given by $Y$. Set

$$
Q_{X}=\left\{g \in G \mid g^{2}=e, g \neq e, \operatorname{dim} X^{g}=k-1\right\},
$$

and let $\left(Q_{X}\right)_{R}$ denote the $(-1)^{k}$-form parameter (see Section 3 ) of $R[G]$ generated by $Q_{X}$. A homomorphism

$$
\mathcal{F}_{\widetilde{X}, R}:\left(\mathbb{Z}\left[\widetilde{G}_{X}\right],(-1)^{k},-,\left(Q_{\widetilde{X}}\right)_{\mathbb{Z}}\right) \longrightarrow\left(R[G],(-1)^{k},-,\left(Q_{X}\right)_{R}\right)
$$


of form rings is naturally defined. Dosing Cappell-Shaneson's group

$$
\Gamma_{2 k}^{h}\left(\mathbb{Z}\left[\widetilde{G}_{X}\right] \rightarrow R[G]\right) \quad(\text { cf. }[2])
$$

with quadratic form parameter, we obtain an abelian group $\Gamma_{(-1)^{k}}\left(\mathcal{F}_{\widetilde{X}, R}\right)_{\mathcal{N}}$.

Let $f^{*} \widetilde{Y}$ denote the induced covering space on $X$ from $\widetilde{Y}$ by $f$ and let

$$
\mathcal{F}_{f^{*} \widetilde{Y}, R}:\left(\mathbb{Z}\left[\widetilde{G}_{Y}\right],(-1)^{k},-,\left(Q_{f * \widetilde{Y}}\right)_{\mathbb{Z}}\right) \longrightarrow\left(R[G],(-1)^{k},-,\left(Q_{X}\right)_{R}\right)
$$

denote the canonical homomorphism.

First, consider the case where $f$ is $k$-connected. Then $\widetilde{G}_{X}=\widetilde{G}_{Y}$ and $\widetilde{X}=$ $f^{*} \widetilde{Y}$. Similarly to [2], one would obtain an element $\sigma_{\mathrm{CS}}(f)$ in $\Gamma_{(-1)^{k}}\left(\mathcal{F}_{\widetilde{X}, R}\right)_{\mathcal{N}}(=$ $\left.\Gamma_{(-1)^{k}}\left(\mathcal{F}_{f^{*} \widetilde{Y}, R}\right)_{\mathcal{N}}\right)$ and $\sigma_{\mathrm{CS}}(f)$ would vanish if and only if $f$ could be converted to an $R$-homology equivalence $f^{\prime}: X^{\prime} \rightarrow Y$ by $G$-surgery on $X_{\text {reg. }}$. Next, consider the case where $f$ is not necessarily $k$-connected. Then we convert $f$ to a degree-one, $k$-connected, $G$-framed map $f^{\prime \prime}: X^{\prime \prime} \rightarrow Y$ by $G$-surgery on $X_{\text {reg. }}$. One would define $\sigma_{\mathrm{CS}}(f)$ in $\Gamma_{(-1)^{k}}\left(\mathcal{F}_{\widetilde{X^{\prime \prime}}, R}\right)_{\mathcal{N}}\left(\right.$ not in $\left.\Gamma_{(-1)^{k}}\left(\mathcal{F}_{\widetilde{X}, R}\right)_{\mathcal{N}}\right)$ to be the element $\sigma_{\mathrm{CS}}\left(f^{\prime \prime}\right)$ as was done in [2]. (Note that $\Gamma_{(-1)^{k}}\left(\mathcal{F}_{\widetilde{X}^{\prime \prime}, R}\right)_{\mathcal{N}}=$ $\left.\Gamma_{(-1)^{k}}\left(\mathcal{F}_{f * \widetilde{Y}, R}\right)_{\mathcal{N}} \cdot\right)$

But this framework defining $\sigma_{\mathrm{CS}}(f)$ is not best for study of surgery problems. To observe it, take a degree-one, $k$-connected, $G$-framed map $f_{0}: X_{0} \rightarrow$ $Y_{0}$ such that $Y_{0}$ is a $2 k$-dimensional homology disk with nontrivial fundamental group, such that the restriction $\partial f_{0}: \partial X_{0} \rightarrow \partial Y_{0}$ is the identity $G$-framed map on $\partial X_{0}$, hence on $\partial Y_{0}$, and such that for some point $x_{0} \in X^{G} \backslash \partial X$, the restriction of $f_{0}$ to a small neighborhood of $x_{0}$ is the identity $G$-framed map. Then we can consider the $G$-connected sum

$$
f_{1}:=f_{0} \#\left(-i d_{Y_{0}} \cup_{\partial} f_{0}\right): X_{0} \#\left(-Y_{0} \cup_{\partial} X_{0}\right) \longrightarrow Y_{0}
$$

at the point $x_{0}\left(=f_{0}\left(x_{0}\right)\right)$. In this situation, we would conjecture

Conjecture 1.1. The equality $\sigma_{\mathrm{CS}}\left(f_{1}\right)=2 \sigma_{\mathrm{CS}}\left(f_{0}\right)$ in $\Gamma_{(-1)^{k}}\left(\mathcal{F}_{\widetilde{X}_{0}, R}\right)_{\mathcal{N}}$ holds.

If we attempt to prove the conjecture within the framework above, we have to take a degree-one, $k$-connected, $G$-framed map $f_{2}: X_{2} \rightarrow Y_{0}$ which is $G$ framed cobordant to $f_{1}$ and realize the abstract object $\sigma_{\mathrm{CS}}\left(f_{1}\right)$ as the concrete one $\sigma_{\mathrm{CS}}\left(f_{2}\right)$. But it is not easy to see what $f_{2}$ is actually, and therefore it is not easy to prove the conjecture within the framework above.

In order to overcome the difficulty, we shall introduce an abelian group $\Gamma_{(-1)^{k}}\left(\mathcal{F}_{\widetilde{X}, R}\right)_{\mathcal{M}}$ as well as canonical homomorphisms

$$
\Gamma_{(-1)^{k}}\left(\mathcal{F}_{\widetilde{X}, R}\right)_{\mathcal{M}} \rightarrow \Gamma_{(-1)^{k}}\left(\mathcal{F}_{f^{*} \widetilde{Y}, R}\right)_{\mathcal{M}} ; \gamma \mapsto \gamma_{\mathbb{Z}\left[\widetilde{G}_{Y}\right]}
$$


and

$$
\Gamma_{(-1)^{k}}\left(\mathcal{F}_{f^{*} \widetilde{Y}, R}\right)_{\mathcal{N}} \rightarrow \Gamma_{(-1)^{k}}\left(\mathcal{F}_{f^{*} \widetilde{Y}, R}\right)_{\mathcal{M}}
$$

In addition, we shall show that the homomorphism

$$
\Gamma_{(-1)^{k}}\left(\mathcal{F}_{f^{*} \widetilde{Y}, R}\right)_{\mathcal{N}} \rightarrow \Gamma_{(-1)^{k}}\left(\mathcal{F}_{f^{*} \widetilde{Y}, R}\right)_{\mathcal{M}}
$$

is an isomorphism (Theorem 3.1). We shall define an $R$-suitable, $G$-framed map in Section 2 which is a slight generalization of a $k$-connected, $G$-framed map. Each $R$-suitable, $G$-framed map $f: X \rightarrow Y$ directly determines a $G$-surgery obstruction $\sigma(f) \in \Gamma_{(-1)^{k}}\left(\mathcal{F}_{\widetilde{X}, R}\right)_{\mathcal{M}}$. We shall show the equality

$$
\sigma(f)_{\mathbb{Z}\left[\widetilde{G}_{Y}\right]}=\sigma_{\mathrm{CS}}(f) \text { in } \Gamma_{(-1)^{k}}\left(\mathcal{F}_{f * \widetilde{Y}, R}\right)_{\mathcal{M}}
$$

In fact, we shall prove the next theorem in this paper.

Theorem 1.1. Let $R$ denote either $\mathbb{Z}$ or $\mathbb{Z}_{(p)}$ for a prime $p$. Let $\boldsymbol{f}=$ $(f, b)$ be a degree-one, $G$-framed map consisting of $f:(X, \partial X) \rightarrow(Y, \partial Y)$ and $b: T(X) \oplus f^{*} \eta \rightarrow f^{*} \xi$. Assume the gap condition (GC) and the following.

(1) The restriction $f^{Q}: X^{Q} \rightarrow Y^{Q}$ is a $\mathbb{Z}_{(q)}$-homology equivalence for every subgroup $\{e\} \neq Q \subseteq G$ having q-power order such that $q$ is a prime not invertible in $R$.

(2) The equality $\chi\left(X^{g}\right)=\chi\left(Y^{g}\right)$ holds for any $g \in G \backslash\{e\}$.

(3) The restriction $\partial f: \partial X \rightarrow \partial Y$ is an R-homology equivalence.

(4) In the case $R=\mathbb{Z}, f^{H}: X^{H} \rightarrow Y^{H}$ is a homology equivalence for every hyperelementary subgroup $\{e\} \neq H \subseteq G$ or $\widetilde{K}_{0}(\mathbb{Z}[G])=0$.

Then following (I) and (II) hold:

(I) There exists an element $\sigma_{\mathrm{CS}}(\boldsymbol{f})$ in $\Gamma_{(-1)^{k}}\left(\mathcal{F}_{f^{*} \widetilde{Y}, R}\right)_{\mathcal{N}}\left(=\Gamma_{(-1)^{k}}\left(\mathcal{F}_{f^{*} \widetilde{Y}, R}\right)_{\mathcal{M}}\right)$ possessing the following properties (i)-(iii):

(i) $\sigma_{\mathrm{CS}}(\boldsymbol{f})$ is an invariant of the $G$-framed cobordism class of $\boldsymbol{f}$ relative to the boundary and the singular set.

(ii) If $f: X \rightarrow Y$ is a one-connected, $R$-homology equivalence then $\sigma_{\mathrm{CS}}(\boldsymbol{f})=$ 0 .

(iii) If $\sigma_{\mathrm{CS}}(\boldsymbol{f})=0$ then one can convert $\boldsymbol{f}$ by $G$-surgery on $X_{\text {reg }}$ to a degree-one, $G$-framed map $\boldsymbol{f}^{\prime}=\left(f^{\prime}, b^{\prime}\right)$ such that $f^{\prime}$ is a $(k-1)$ connected, R-homology equivalence, where $f^{\prime}:\left(X^{\prime}, \partial X^{\prime}\right) \rightarrow(Y, \partial Y)$ and $b^{\prime}: T\left(X^{\prime}\right) \oplus f^{\prime *} \eta \rightarrow f^{\prime *} \xi$. 
(II) If $\boldsymbol{f}$ is R-suitable (see Section 2) then there exists an element $\sigma(\boldsymbol{f})$ in $\Gamma_{(-1)^{k}}\left(\mathcal{F}_{\widetilde{X}, R}\right)_{\mathcal{M}}$ having the following properties (iv)-(v):

(iv) If $\sigma(\boldsymbol{f})=0$ and $f: X \rightarrow Y$ is $\ell$-connected for some integer $\ell$ such that $1 \leq \ell \leq k-1$ then one can convert $f$ by $(k-1)$ - and $k$-dimensional $G$-surgery on $X_{\text {reg }}$ to a degree-one $G$-framed map $f^{\prime}=\left(f^{\prime}, b^{\prime}\right)$ such that $f^{\prime}: X^{\prime} \rightarrow Y$ is an $\ell$-connected, R-homology equivalence, where $f^{\prime}:\left(X^{\prime}, \partial X^{\prime}\right) \rightarrow(Y, \partial Y)$ and $b^{\prime}: T\left(X^{\prime}\right) \oplus{f^{\prime *}}^{*} \rightarrow f^{\prime *} \xi$.

(v) The equality $\sigma(\boldsymbol{f})_{\mathbb{Z}\left[\widetilde{G}_{Y}\right]}=\sigma_{\mathrm{CS}}(\boldsymbol{f})$ holds in $\Gamma_{(-1)^{k}}\left(\mathcal{F}_{f^{*} \widetilde{Y}, R}\right)_{\mathcal{M}}$.

We shall verify Conjecture 1.1 in a general setting: namely, as Theorem 1.2 below. So as to take a geometric connected sum of $\boldsymbol{f}, \boldsymbol{f}^{\prime}$ and $\boldsymbol{i} \boldsymbol{d}_{Y}$, we invoke the next.

Context 1.1. Let $\boldsymbol{f}=(f, b)$ and $\boldsymbol{f}^{\prime}=\left(f^{\prime}, b^{\prime}\right)$, where $f:(X, \partial X) \rightarrow$ $(Y, \partial Y), b: \varepsilon_{X}(\mathbb{R}) \oplus T(X) \oplus f^{*} \eta \rightarrow f^{*} \tau, f^{\prime}:\left(X^{\prime}, \partial X^{\prime}\right) \rightarrow(Y, \partial Y)$, and $b^{\prime}: \varepsilon_{X^{\prime}}(\mathbb{R}) \oplus T\left(X^{\prime}\right) \oplus{f^{\prime *}}^{*} \rightarrow f^{\prime *} \tau$, be degree-one, $G$-framed maps such that $\tau=\varepsilon_{Y}(\mathbb{R}) \oplus T(Y) \oplus \eta, \partial X^{\prime}=\partial Y$ and $\left.\boldsymbol{f}^{\prime}\right|_{\partial X^{\prime}}=\boldsymbol{i} \boldsymbol{d}_{\partial Y}$.

Define $\Delta X^{\prime}=(-Y) \cup_{\partial(-Y)} X^{\prime}$. If $X^{\prime}=Y$ then $\Delta X^{\prime}$ is the double of $Y$. As in Section 3 of [8], we define the $G$-framed map $\Delta f^{\prime}=\left(\Delta f^{\prime}, \Delta b^{\prime}\right)$ with $\Delta f^{\prime}: \Delta X^{\prime} \rightarrow Y$ and $\Delta b^{\prime}: \varepsilon_{\Delta X^{\prime}}(\mathbb{R}) \oplus T\left(X^{\prime}\right) \oplus\left(\Delta f^{\prime}\right)^{*} \eta \rightarrow\left(\Delta f^{\prime}\right)^{*} \tau$. Let $y_{0} \in Y^{G}$ be a base point located in the interior of $Y$ and $V_{y_{0}}$ a $G$-linear slice neighborhood of $y_{0}$ with a $G$-invariant inner product in $Y$. Let $x_{1} \in f^{-1}\left(y_{0}\right)$ and $G \times_{H} V$ a $G$-tubular neighborhood of $G x_{1}$ in $X$ such that $H=G_{x_{1}}$ and $V$ is an $H$-linear slice neighborhood of $x_{1}$. Suppose $\left.f\right|_{V}: V \rightarrow V_{y_{0}}$ is an orientation-preserving linear isomorphism. Then, we define the connected sum $X \#_{G, x_{1}}\left(G \times_{H} \Delta X^{\prime}\right)$ $\left(=X^{\prime \prime}\right.$ say $)$ by

$$
X^{\prime \prime}=\left(X \backslash G \cdot \operatorname{Interior}\left(D_{x_{1}}\right)\right) \cup_{\partial G \cdot D_{x_{1}}}\left(G \times_{H}\left(\Delta X^{\prime} \backslash \operatorname{Interior}\left(D_{y_{0}}\right)\right)\right),
$$

where $D_{x_{1}}$ and $D_{y_{0}}$ are the unit disks of $V$ and $V_{y_{0}}$ concerned with some $H$-invariant inner-product and some $G$-invariant inner-product, respectively. Allow us to regard $\left.f\right|_{V}: V \rightarrow V_{y_{0}}$ as the identity map and suppose $\left.b\right|_{V}=$ $i d_{\left.\tau\right|_{V_{0}}}$. Then we construct the $G$-connected sum

$$
\boldsymbol{f} \#_{G, x_{1}}\left(G \times_{H} \Delta \boldsymbol{f}^{\prime}\right)=\left(f \#_{G, x_{1}}\left(G \times_{H} \Delta f^{\prime}\right), b \#_{G, x_{1}}\left(G \times_{H} \Delta b^{\prime}\right)\right)
$$

consisting of $f \#_{G, x_{1}}\left(G \times_{H} \Delta f^{\prime}\right): X \#_{G, x_{1}}\left(G \times_{H} \Delta X^{\prime}\right) \rightarrow Y\left(=f^{\prime \prime}\right.$ say $)$ and $b \#_{G, x_{1}}\left(G \times_{H} \Delta b^{\prime}\right): \varepsilon_{X^{\prime \prime}}(\mathbb{R}) \oplus T\left(X^{\prime \prime}\right) \oplus f^{\prime \prime *} \eta \rightarrow f^{\prime \prime *} \tau$, and the $G$-connected sum 
$\boldsymbol{f} \#_{G, x_{1}}\left(G \times_{H}\left(-\Delta \boldsymbol{f}^{\prime}\right)\right)$ (for the details see Section 3 of [8]). There is canonically defined an induction homomorphism

$$
\operatorname{Ind}_{(\widehat{H}, H)}^{(\widehat{G}, G)}: \Gamma_{(-1)^{k}}\left(\mathcal{F}_{\left(\operatorname{Res}_{H}^{G} f\right) * \operatorname{Res}_{H}^{G} Y, R}\right)_{\mathcal{M}} \rightarrow \Gamma_{(-1)^{k}}\left(\mathcal{F}_{f * \widetilde{Y}, R}\right)_{\mathcal{M}}
$$

(see Section 5, the last paragraph in Proof of Theorem 1.2). The next theorem describes the geometric connected sum in terms with the algebraic induction homomorphism.

Theorem 1.2. $\quad$ Let $R$ denote either $\mathbb{Z}$ or $\mathbb{Z}_{(p)}$ for a prime $p$, and let $\boldsymbol{f}$ and $\boldsymbol{f}^{\prime}$ be degree-one, one-connected, G-framed maps in Context 1.1 satisfying the gap condition $(G C)$ and fulfilling (1)-(4) in Theorem 1.1. Suppose that $Y$ and $\partial Y$ are $R$-homology equivalent to $D^{n}$ and $S^{n-1}$ respectively, and the induced homomorphism $\pi_{1}(\partial Y) \rightarrow \pi_{1}(Y)$ by the inclusion map is an isomorphism. Let $y_{0} \in Y, V_{y_{0}}, x_{1} \in X$ and $V$ be also as in Context 1.1. Then

$$
\sigma_{\mathrm{CS}}\left(\boldsymbol{f} \#_{G, x_{1}}\left(G \times_{H}\left(\Delta \boldsymbol{f}^{\prime}\right)\right)\right)=\sigma_{\mathrm{CS}}(\boldsymbol{f})+\operatorname{Ind}_{(\widehat{H}, H)}^{(\widehat{G}, G)} \sigma_{\mathrm{CS}}\left(\operatorname{Res}_{H}^{G} \boldsymbol{f}^{\prime}\right),
$$

where $\widehat{G}=\widetilde{G}_{Y}$ and $\widehat{H}=\pi_{1}\left(E G \times_{H} Y\right)$.

Applying our equivariant surgery theory, we can decide which closed smooth manifolds can occur as the $G$-fixed point sets of smooth actions on spheres for a perfect or nilpotent Oliver group $G$, which is discussed in [8].

The rest of the paper is organized as follows. In Section 2 , we define a $G$-framed normal map $f$, the notion of $R$-suitable and the $(-1)^{k}$-quadratic module $\alpha(\boldsymbol{f})$ associated with $R$-suitable $\boldsymbol{f}$. In Section 3, we observe basic properties of $\lambda$-quadratic modules over $\mathcal{F}$, define the groups $\Gamma_{\lambda}(\mathcal{F})_{\mathcal{M}}$ and $\Gamma_{\lambda}(\mathcal{F})_{\mathcal{N}}$, and discuss basic properties of the groups. In particular, we refer to an isomorphism from $\Gamma_{\lambda}(\mathcal{F})_{\mathcal{N}}$ to the Bak group (Theorem 3.2). In Section 4, we define the element $\sigma(\boldsymbol{f})$ for $R$-suitable $\boldsymbol{f}$ and give a cobordism-invariance theorem of $\sigma(\boldsymbol{f})_{\mathbb{Z}\left[\widetilde{G}_{Y}\right]}$ (Theorem 4.2). In Section 5, we define $\sigma_{\mathrm{CS}}(\boldsymbol{f})$ and prove Theorems 1.1 and 1.2 .

\section{$\S 2 . \quad R$-suitable $G$-framed Maps and Associated Data}

Let $G$ be a finite group, $R$ a commutative ring with unit, and $X, Y$ compact, connected, oriented, smooth $G$-manifolds. Unless otherwise specified, we invoke the gap condition (GC) on $X$. In the case where a relevant $G$-map $f: X \rightarrow Y$ is clear from the context, we denote $f^{*} \widetilde{Y}$ by $\widehat{X}$. Let $\widetilde{G}$ and $\widehat{G}$ denote $\widetilde{G}_{X}$ and $\widetilde{G}_{Y}$, respectively. Then $\widetilde{G}$ acts on $\widetilde{X}$, and $\widehat{G}$ acts on $\widetilde{Y}$ as well 
as $\widehat{X}$. Note that if $Y^{G} \neq \varnothing$, then $\widehat{G}$ is a semidirect product of $G$ with $\pi_{1}(Y)$, i.e. $\widehat{G}=G \ltimes \pi_{1}(Y)$. Since the $G$-action on $X$ satisfies the gap condition, the $\widetilde{G}$-action on $\widetilde{X}$ and the $\widehat{G}$-action on $\widehat{X}$ both fulfill the gap condition. For a $G$-map $f: X \rightarrow Y$, let $M_{f}$ and $\widetilde{f}$ denote the mapping cylinder of $f$ and the map $\widetilde{X} \rightarrow \widetilde{Y}$ covering $f$, respectively. The map $\widetilde{f}$ is regarded as a $\widetilde{G}$-map.

A $G$-framed map $\boldsymbol{f}=(f, b)$ is a pair consisting of a $G$-map $f:(X, \partial X) \rightarrow$ $(Y, \partial Y)$ and a $G$-vector bundle isomorphism $b: T(X) \oplus f^{*} \eta \rightarrow f^{*} \xi$ covering the identity map on $X$ for some $G$-vector bundles $\eta$ and $\xi$ over $Y$. If $f:(X, \partial X) \rightarrow$ $(Y, \partial Y)$ is of degree one, then we say that $f$ is of degree one. Similarly, if $f: X \rightarrow Y$ is an $R$-homology equivalence (resp. homotopy equivalence), then $f$ is said to be an $R$-homology equivalence (resp. homotopy equivalence).

Let $p$ be a prime and $\boldsymbol{f}=(f, b)$ a degree-one, one-connected, $G$-framed map such that $X$ is of even dimension $n=2 k \geq 6$, where $f:(X, \partial X) \rightarrow(Y, \partial Y)$. Here the one-connectivity means that $f_{\#}: \pi_{1}(X) \rightarrow \pi_{1}(X)$ is surjective. Set

$$
\Pi(f)=\pi_{k+1}\left(M_{\tilde{f}}, \widetilde{X}\right) .
$$

Then $\Pi(f)$ is an abelian group by $k \geq 3$, moreover a $\mathbb{Z}[\widetilde{G}]$-module since $\tilde{X}$ is one-connected. Each element $x$ of $\Pi(f)$ is represented by a commutative diagram $\operatorname{diag}(x)$ :

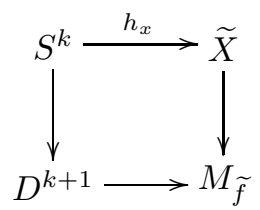

with an immersion $h_{x}$. By using $h_{x}: S^{k} \rightarrow \widetilde{X}$ for each $x \in \Pi(f)$, we get the ordinary intersection form

$$
\varphi_{e}: \Pi(f) \times \Pi(f) \rightarrow \mathbb{Z}
$$

and hence the equivariant intersection form

$$
\varphi: \Pi(f) \times \Pi(f) \rightarrow \mathbb{Z}[\widetilde{G}]
$$

given by

$$
\varphi\left(x, x^{\prime}\right)=\sum_{a \in \widetilde{G}} \varphi_{e}\left(x, a^{-1} x^{\prime}\right) a
$$

for $x, x^{\prime} \in \Pi(f)$. Recall that the immersion $h_{x}$ extends to $D^{k+1} \rightarrow M_{\widetilde{f}}$ in $\operatorname{diag}(x)$. By virtue of the bundle datum $b$, the vector bundle $T\left(S^{k}\right) \oplus \nu\left(S^{k} \leftrightarrow \widetilde{X}\right)$ is stably trivial. By Hirsch's immersion-classification theorem, the ingredient 
$h_{x}$ of $\operatorname{diag}(x)$ can be chosen so that the normal bundle is trivial, moreover such an immersion is uniquely determined up to regular homotopies. Thus, we obtain the self-intersection form

$$
\mu_{e}: \Pi(f) \rightarrow \mathbb{Z} /\left(1-(-1)^{k}\right) .
$$

Let $\widetilde{G}(2)$ denote the set of all elements in $\widetilde{G}$ of order 2 . If $a \in \widetilde{G}(2)$ satisfies $\operatorname{dim} \widetilde{X}^{a} \leq k-2$, then by counting the number of appropriately signed selfintersection points of the immersion $p_{\langle a\rangle} \circ h_{x}$ set in a general position which do not arise from self-intersection points of $h_{x}$, we obtain the self-intersection form

$$
\mu_{a}: \Pi(f) \rightarrow \mathbb{Z} /\left(1-(-1)^{k} w(a)\right),
$$

where $p_{\langle a\rangle}$ is the projection

$$
\widetilde{X} \backslash \widetilde{X}^{a} \rightarrow\left(\tilde{X} \backslash \widetilde{X}^{a}\right) /\langle a\rangle,
$$

and $w$ is the orientation homomorphism $\widetilde{G} \rightarrow\{1,-1\}$ associated with $\widetilde{X}$. Set

$$
\widetilde{Q}=Q_{\widetilde{X}}\left(=\left\{a \in \widetilde{G}(2) \mid \operatorname{dim} \widetilde{X}^{a}=k-1\right\}\right) .
$$

Let $R$ be a commutative ring with unit, and let $(\widetilde{Q})_{R}$ denote the $(-1)^{k}$-form parameter of $R[\widetilde{G}]$ generated by $\widetilde{Q}$ : namely,

$$
(\widetilde{Q})_{R}=\left\langle a-(-1)^{k} \bar{a} \mid a \in \widetilde{G}\right\rangle_{R}+\langle a \mid a \in \widetilde{Q}\rangle_{R} \subseteq R[\widetilde{G}]
$$

Set

$$
\begin{aligned}
\widetilde{G}(2)_{s} & =\left\{a \in \widetilde{G}(2) \mid w(a)=(-1)^{k}\right\}, \\
\widetilde{G}(2)_{q} & =\left\{a \in \widetilde{G}(2) \mid w(a)=-(-1)^{k}\right\} .
\end{aligned}
$$

We decompose $\widetilde{G}$ to a disjoint union:

$$
G=\{e\} \amalg \widetilde{G}(2)_{s} \amalg \widetilde{G}(2)_{q} \amalg C \amalg C^{-1},
$$

where $C^{-1}=\left\{a^{-1} \mid a \in C\right\}$. We set

$$
\widetilde{\mathcal{Q}}=\{e\} \amalg \widetilde{G}(2)_{s} \amalg\left(\widetilde{G}(2)_{q} \backslash \widetilde{Q}\right) \amalg C .
$$

For $a \in \widetilde{G}$, we set

$$
R_{a}= \begin{cases}R /\left(1-(-1)^{k}\right) R & (a=e), \\ R & \left(a \in \widetilde{G}(2)_{s}\right), \\ R / 2 R & \left(a \in \widetilde{G}(2)_{q} \backslash \widetilde{Q}\right), \\ R & \text { (otherwise). }\end{cases}
$$


Then $R[\widetilde{G}] /(\widetilde{Q})_{R}$ is identified with $\bigoplus_{a \in \widetilde{\mathcal{Q}}} R_{a}$. Thus a map $\gamma: \Pi(f) \rightarrow R[\widetilde{G}] /$ $(\widetilde{Q})_{R}$ can be understood as a formal sum of $\gamma_{a}: \Pi(f) \rightarrow R_{a}, a \in \widetilde{\mathcal{Q}}$. We define the equivariant self-intersection form

$$
\mu: \Pi(f) \rightarrow \mathbb{Z}[\widetilde{G}] /(\widetilde{Q})_{\mathbb{Z}}
$$

by using $\mu_{e}, \mu_{a}$ above for $a \in \widetilde{G}(2) \backslash \widetilde{Q}$ and

$$
\mu_{a}(x)=\varphi_{e}\left(x, a^{-1} x\right)
$$

for $a \in C$.

We shall define an element $\sigma(f)$ which will be an obstruction to converting $f$ by $G$-surgery on the regular set of $X$ to a $\mathbb{Z}_{(p)}$-homology equivalence $f^{\prime}=$ $\left(f^{\prime}, b^{\prime}\right)$ consisting of $f^{\prime}:\left(X^{\prime}, \partial X\right) \rightarrow(Y, \partial Y)$ and $b^{\prime}: T\left(X^{\prime}\right) \oplus f^{\prime *} \eta \rightarrow f^{\prime *} \xi$. For the goal, we have the following necessary conditions derived from the Smith theory:

(S1) $f^{P}: X^{P} \rightarrow Y^{P}$ is a $\mathbb{Z}_{(p)}$-homology equivalence for any $p$-subgroup $P \neq\{e\}$,

(S2) $\chi\left(X^{g}\right)=\chi\left(Y^{g}\right)$ for all $g \in G \backslash\{e\}$.

The element $\sigma(\boldsymbol{f})$ will be defined for $\boldsymbol{f}$ such that

(S3) $f_{*}: H_{i}\left(X ; \mathbb{Z}_{(p)}\right) \rightarrow H_{i}\left(Y ; \mathbb{Z}_{(p)}\right)$ are isomorphisms for all $i<k$; and the canonical map

$$
\kappa: \mathbb{Z}_{(p)} \otimes \Pi(f) \rightarrow\left(H_{k+1}\left(M_{\tilde{f}}, \tilde{X} ; \mathbb{Z}_{(p)}\right) \rightarrow\right) H_{k+1}\left(M_{f}, X ; \mathbb{Z}_{(p)}\right)
$$

is an epimorphism,

Since $f$ is of degree one, the $\mathbb{Z}_{(p)}[G]$-module $H_{k+1}\left(M_{f}, X ; \mathbb{Z}_{(p)}\right)$ is identified with

$$
K_{k}\left(X ; \mathbb{Z}_{(p)}\right)=\operatorname{Ker}\left[f_{*}: H_{k}\left(X ; \mathbb{Z}_{(p)}\right) \rightarrow H_{k}\left(Y ; \mathbb{Z}_{(p)}\right)\right]
$$

via the canonical homomorphism. Note that if $f$ is $k$-connected, then (S3) is automatically satisfied. In order to avoid difficulties caused by the existence of boundaries, we invoke the additional condition:

(S4) $\partial f: \partial X \rightarrow \partial Y$ is a $\mathbb{Z}_{(p)}$-homology equivalence.

For a prime $p$, a $G$-framed map $f=(f, b)$ is said to be $\mathbb{Z}_{(p)}$-suitable if $f$ is one-connected, of degree one, and satisfies (S1)-(S4). If $\boldsymbol{f}=(f, b)$ is $\mathbb{Z}_{(p)}$-suitable, then $K_{k}\left(X ; \mathbb{Z}_{(p)}\right)$ is $\mathbb{Z}_{(p)}[G]$-free. 
A $G$-framed map $f=(f, b)$ is said to be $\mathbb{Z}$-suitable if it is $\mathbb{Z}_{(p)}$-suitable for every prime $p$ and

$(\mathrm{S} 5) K_{k}(X ; \mathbb{Z})=\operatorname{Ker}\left[f_{*}: H_{k}(X ; \mathbb{Z}) \rightarrow H_{k}(Y ; \mathbb{Z})\right]$ is stably $\mathbb{Z}[G]$-free.

If $\boldsymbol{f}=(f, b)$ is $\mathbb{Z}$-suitable, then

$\left(\mathrm{S} 3^{\prime}\right) f_{*}: H_{i}(X ; \mathbb{Z}) \rightarrow H_{i}(Y ; \mathbb{Z})$ are isomorphisms for all $i<k$; and the canonical map

$$
\kappa: \Pi(f) \rightarrow\left(H_{k+1}\left(M_{\widetilde{f}}, \tilde{X} ; \mathbb{Z}\right) \rightarrow\right) H_{k+1}\left(M_{f}, X ; \mathbb{Z}\right)
$$

is an epimorphism,

$\left(\mathrm{S} 4^{\prime}\right) \partial f: \partial X \rightarrow \partial Y$ is a $\mathbb{Z}$-homology equivalence.

Let $R$ denote either $\mathbb{Z}$ or $\mathbb{Z}_{(p)}$ for some prime $p$. If $\boldsymbol{f}=(f, b)$ is $R$-suitable, then we have the equivariant intersection form

$$
\underline{\varphi}: K_{k}(X ; R) \times K_{k}(X ; R) \rightarrow R[G]
$$

compatible with $\varphi$ above: namely,

$$
\underline{\varphi}\left(\kappa(x), \kappa\left(x^{\prime}\right)\right)=\mathcal{F}(\varphi(x, y))
$$

for $x, x^{\prime} \in \Pi(f)$, where $\mathcal{F}: \mathbb{Z}[\widetilde{G}] \rightarrow R[G]$ is the canonical homomorphism. Since $\varphi$ is essentially the Poincaré pairing, $\underline{\varphi}$ is nonsingular, namely the adjoint map

$$
\Phi: K_{k}(X ; R) \rightarrow \operatorname{Hom}_{R[G]}\left(K_{k}(X ; R), R[G]\right)
$$

given by $\Phi(x)(y)=\varphi(x, y)$ for $x, y \in K_{k}(X ; R)$ is an isomorphism. Note that in the special case where $f$ is $k$-connected, $R[G] \otimes_{\mathbb{Z}[\widetilde{G}]} \Pi(f) \cong K_{k}(X ; R)$; and the equivariant self-intersection form

$$
\underline{\mu}: K_{k}(X ; R) \rightarrow R[G] /(Q)_{R}
$$

is induced from $\mu$, where

$$
Q=\left\{g \in G(2) \mid \operatorname{dim} X^{g}=k-1\right\} .
$$

Definition 2.1. Let $f=(f, b)$ be an $R$-suitable $G$-framed map as above. Then we call the datum

$$
\alpha(\boldsymbol{f})=\left(\kappa: \Pi(f) \rightarrow K_{k}(X ; R), \varphi, \mu, \underline{\varphi}\right)
$$

the $(-1)^{k}$-quadratic module over $\mathcal{F}_{X, R}$ associated with $\boldsymbol{f}$. 
The next lemma follows from standard arguments (see $[10,5]$ ).

Lemma 2.1. $\quad$ Let $R$ denote $\mathbb{Z}$ or $\mathbb{Z}_{(p)}$. Let $\ell$ be an integer such that $1 \leq \ell \leq k-1$. Let $X$ be a compact, oriented, smooth $G$-manifold of dimension $n=2 k \geq 6$ satisfying the gap condition (GC) and let $f=(f, b)$ be an $\ell$ connected, $R$-suitable $G$-framed map consisting of $f:(X, \partial X) \rightarrow(Y, \partial Y)$ and $b: T(X) \oplus f^{*} \eta \rightarrow f^{*} \xi$. If there exists a finitely generated $\mathbb{Z}[\widetilde{G}]$-submodule $L \subset \Pi(f)$ such that

(1) $\varphi(L, L)=\{0\}$ and $\mu(L)=\{0\}$,

$(2)\langle\kappa(L)\rangle_{R}$ is a stably free $\mathbb{Z}[\widetilde{G}]$-direct summand of $K_{k}(X ; R)$, and

(3) the submodule

$$
\left\{x \in K_{k}(X ; R) \mid \underline{\varphi}\left(x, \kappa\left(x^{\prime}\right)\right)=0 \text { for all } x^{\prime} \in L\right\}
$$

coincides with $\langle\kappa(L)\rangle_{R}$,

then one can perform $G$-surgery on $X_{\mathrm{reg}}$ of dimension $k-1$ and $k$ so that the resulting $f^{\prime}=\left(f^{\prime}, b^{\prime}\right)$ is an $\ell$-connected, $R$-homology equivalence.

The submodule $L$ above is referred to as a pre-Lagrangian (or presubkernel) of $\alpha(\boldsymbol{f})$

\section{§3. Extended Cappell-Shaneson's Group}

Let $A$ be a ring with unit. A map - : $A \rightarrow A$ is called an involution if $\overline{1}=1, \overline{a+b}=\bar{a}+\bar{b}$ and $\overline{a b}=\bar{b} \bar{a}$ are satisfied for all $a, b \in A$. A ring homomorphism $\psi: A \rightarrow A^{\prime}$ is said to be locally epic if for arbitrary (finitely many) elements $a_{1}^{\prime}, \ldots, a_{m}^{\prime}$ of $A^{\prime}$, there exists a unit $u \in A^{\prime}$ such that all $u a_{1}^{\prime}, \ldots, u a_{m}^{\prime}$ lie in $\psi(A)$. If moreover one can take the $u$ above in $\psi(A)$, then $\psi$ is said to be strongly locally epic. For example, the canonical homomorphism $\mathbb{Z} \rightarrow \mathbb{Z}_{(p)}$ is strongly locally epic. Unless otherwise stated, we assume each module (over $A$ say) is finitely generated (over $A$ ). Let $M$ and $M^{\prime}$ be modules over $A$ and $A^{\prime}$, respectively. A $\psi$-homomorphism $h: M \rightarrow M^{\prime}$ is said to be locally epic if for arbitrary $x_{1}^{\prime}, \ldots, x_{m}^{\prime}$ in $M^{\prime}$, there exists a unit $u \in A^{\prime}$ such that all $u x_{1}^{\prime}, \ldots, u x_{m}^{\prime}$ lie in $h(M)$.

Let $\lambda=1$ or -1 . An additive subgroup $\Lambda$ of $A$ is called a $\lambda$-form parameter if the following conditions are satisfied:

(1) $\{a-\lambda \bar{a} \mid a \in A\} \subseteq \Lambda \subseteq\{a \in A \mid a=-\lambda \bar{a}\}$, 
(2) $a \Lambda \bar{a} \subseteq \Lambda$ for all $a \in A$.

The datum $(A,-, \lambda, \Lambda)$ is referred to as a form ring. A homomorphism (or morphism)

$$
(A,-, \lambda, \Lambda) \rightarrow\left(A^{\prime},-, \lambda, \Lambda^{\prime}\right)
$$

of form rings is a ring homomorphism $\psi: A \rightarrow A^{\prime}$ such that $\psi$ preserves the involution and the form parameter: namely, $\overline{\psi(a)}=\psi(\bar{a})$ for all $a \in A$ and $\psi(\Lambda) \subseteq \Lambda^{\prime}$

Let $\mathcal{F}:(A,-, \lambda, \Lambda) \rightarrow(\underline{A},-, \lambda, \underline{\Lambda})$ be a homomorphism such that $\mathcal{F}: A \rightarrow$ $\underline{A}$ is locally epic.

A $\lambda$-quadratic module $\alpha$ over $\mathcal{F}$ is a tuple $(\kappa: H \rightarrow \underline{H}, \varphi, \mu, \underline{\varphi})$ such that

(1) $H$ is a finitely generated $A$-module,

(2) $\underline{H}$ is a finitely generated stably free $\underline{A}$-module,

(3) $\kappa$ is a locally epic $\mathcal{F}$-homomorphism,

(4) $\varphi: H \times H \rightarrow A$ is a biadditive map,

(5) $\mu: H \rightarrow A / \Lambda$ is a map,

(6) $\underline{\varphi}: \underline{H} \times \underline{H} \rightarrow \underline{A}$ is a biadditive map,

satisfying

(Q1) $\varphi\left(a x, a^{\prime} x^{\prime}\right)=a^{\prime} \varphi\left(x, x^{\prime}\right) \bar{a}$,

(Q2) $\varphi\left(x, x^{\prime}\right)=\lambda \overline{\varphi\left(x^{\prime}, x\right)}$,

(Q3) $\varphi(x, x)=\widetilde{\mu(x)}+\lambda \widetilde{\widetilde{\mu(x)}}$,

(Q4) $\mu\left(x+x^{\prime}\right)-\mu(x)-\mu\left(x^{\prime}\right) \equiv \varphi\left(x, x^{\prime}\right) \bmod \Lambda$,

(Q5) $\mu(a x)=a \mu(x) \bar{a}$,

(Q6) the adjoint map

$$
\begin{gathered}
\Phi: \underline{H} \rightarrow \operatorname{Hom}_{B}(\underline{H}, B) ; \\
\Phi(y)\left(y^{\prime}\right)=\underline{\varphi}\left(y, y^{\prime}\right)
\end{gathered}
$$

is bijective, 
(Q7) $\underline{\varphi}\left(\kappa(x), \kappa\left(x^{\prime}\right)\right)=\mathcal{F}\left(\varphi\left(x, x^{\prime}\right)\right)$,

for all $a, a^{\prime} \in A, x, x^{\prime} \in H, y, y^{\prime} \in \underline{H}$.

A typical example of a $\lambda$-quadratic module over $\mathcal{F}$ is the $\lambda$-hyperbolic plane

$$
\boldsymbol{H}(A)=\left(\gamma: H \rightarrow \underline{H}, \varphi_{0}, \mu_{0}, \underline{\varphi_{0}}\right)
$$

which is given as follows:

(1) $H$ is a free $A$-module with basis $\left\{x_{1}, x_{2}\right\}$ and $\underline{H}$ is a free $\underline{A}$-module with basis $\left\{y_{1}, y_{2}\right\}$

(2) $\gamma\left(x_{1}\right)=y_{1}, \gamma\left(x_{2}\right)=y_{2}$,

(3) $\varphi_{0}\left(x_{1}, x_{1}\right)=0, \varphi_{0}\left(x_{1}, x_{2}\right)=1, \varphi_{0}\left(x_{2}, x_{2}\right)=0$,

(4) $\mu_{0}\left(x_{1}\right)=0, \mu_{0}\left(x_{2}\right)=0$,

(5) $\underline{\varphi_{0}}\left(y_{1}, y_{1}\right)=0, \underline{\varphi_{0}}\left(y_{1}, y_{2}\right)=1, \underline{\varphi_{0}}\left(y_{2}, y_{2}\right)=0$.

A $\lambda$-quadratic module over $\mathcal{F}$ isomorphic to

$$
\boldsymbol{H}\left(A^{m}\right)=\boldsymbol{H}(A) \perp \cdots \perp \boldsymbol{H}(A) \quad \text { (the orthogonal sum) }
$$

is called a $\lambda$-hyperbolic module.

Let $\alpha=(\kappa: H \rightarrow \underline{H}, \varphi, \mu, \underline{\varphi})$ be a $\lambda$-quadratic module over $\mathcal{F}$. We mean by $-\alpha$ the $\lambda$-quadratic module $(\kappa: H \rightarrow \underline{H},-\varphi,-\mu,-\underline{\varphi})$ over $\mathcal{F}$. A finitely generated $A$-submodule $L$ of $H$ is called a pre-Lagrangian (or presubkernel) if the following conditions are satisfied:

(1) $\varphi(L, L)=\{0\}$ and $\mu(L)=\{0\}$.

(2) $\langle\kappa(L)\rangle_{A}$ is a stably free $\underline{A}$-direct summand of $\underline{H}$.

(3) The submodule $\left\{y \in \underline{H} \mid \underline{\varphi}\left(y, y^{\prime}\right)=0\left(\forall y^{\prime} \in \kappa(L)\right)\right\}$ coincides with $\langle\kappa(L)\rangle_{\underline{A}}$.

A $\lambda$-quadratic module over $\mathcal{F}$ is called a null module if it admits a preLagrangian. Clearly, a $\lambda$-hyperbolic module is a null module.

Lemma 3.1. Let $\alpha=(\kappa: H \rightarrow \underline{H}, \varphi, \mu, \varphi)$ be a $\lambda$-quadratic module over $\mathcal{F}$ and $K$ an $A$-submodule of $H$ such that $\kappa(\bar{H})=\kappa(K)$. Then $\alpha \perp-\beta$ is a null module, where $\beta=\left(\left.\kappa\right|_{K}: K \rightarrow \underline{H},\left.\varphi\right|_{K \times K},\left.\mu\right|_{K}, \underline{\varphi}\right)$ and $\perp$ stands for the orthogonal sum. 
Proof. The submodule $L=\{(x, x) \in H \oplus K \mid x \in K\}$ is a pre-Lagrangian of $\alpha \perp-\beta$.

Corollary 3.1 cf. [2, Lemma 1.1]. For an arbitrary $\lambda$-quadratic module $\alpha$ over $\mathcal{F}, \alpha \perp-\alpha$ is a null module.

Lemma 3.2 cf. [2, Lemma 1.2]. For each $\lambda$-quadratic module $\alpha=(\kappa$ : $H \rightarrow \underline{H}, \varphi, \mu, \underline{\varphi})$ over $\mathcal{F}$, there exists a $\lambda$-quadratic module $\alpha^{\prime}=\left(\kappa^{\prime}: H^{\prime} \rightarrow\right.$ $\left.\underline{H}, \varphi^{\prime}, \mu^{\prime}, \underline{\varphi}\right)$ such that $H^{\prime}$ is a free A-module and $\alpha \perp-\alpha^{\prime}$ is a null module.

Proof. Since $H$ is finitely generated over $A$, there exists an $A$-epimorphism $f: H^{\prime} \rightarrow H$ such that $H^{\prime}$ is a finitely generated free $A$-module. We define the ingredients of $\alpha^{\prime}$ by setting $\kappa^{\prime}=\kappa \circ f, \varphi^{\prime}=\varphi \circ(f \times f), \mu^{\prime}=\mu \circ f$. Then $L=\left\{(f(x), x) \in H \oplus H^{\prime} \mid x \in H^{\prime}\right\}$ is a pre-Lagrangian of $\alpha \perp-\alpha^{\prime}$.

We say that $\lambda$-quadratic modules $\alpha$ and $\alpha^{\prime}$ over $\mathcal{F}$ are equivalent and write $\alpha \sim \alpha^{\prime}$ if there exists a null module $\beta$ such that $\alpha \perp-\alpha^{\prime} \perp \beta$ is a null module.

The next lemma is significant for understanding surgery theory.

Lemma 3.3 cf. [2, Lemma 1.3]. A $\lambda$-quadratic module $\alpha$ over $\mathcal{F}$ is equivalent to 0 if and only if there exists a $\lambda$-hyperbolic module $\boldsymbol{H}\left(A^{m}\right)$ such that $\alpha \perp \boldsymbol{H}\left(A^{m}\right)$ is a null module.

Proof. This follows from the arguments in the proof of [2, Lemma 1.3].

Let $\mathcal{M}$ denote the category of $\lambda$-quadratic modules over $\mathcal{F}$ and let $\mathcal{N}$ denote the full subcategory of $\mathcal{M}$ consisting of $\alpha=(\kappa: H \rightarrow \underline{H}, \varphi, \mu, \underline{\varphi})$ such that the induced map $H_{\underline{A}} \rightarrow \underline{H}$ from $\kappa$ is an isomorphism, where $H_{\underline{A}}=$ $\underline{A} \otimes_{A} H$. It is clear that $\lambda$-hyperbolic modules belong to $\mathcal{N}$. Each object $\alpha=(\kappa: H \rightarrow \underline{H}, \varphi, \mu, \underline{\varphi})$ in $\mathcal{N}$ provides a $\lambda$-quadratic map $\underline{\mu}: \underline{H} \rightarrow \underline{A} / \underline{\Lambda}$ such that $\mu(\kappa(x))=[\mathcal{F}(\widetilde{\mu(x)}))]$ for $x \in H$, where $\widetilde{\mu(x)} \in A$ is a lifting of $\mu(x)$. Let $\mathcal{C}=\overline{\mathcal{M}}$ or $\mathcal{N}$ and let $\Gamma_{\lambda}(\mathcal{F})_{\mathcal{C}}$ denote the set of all equivalence classes of $\lambda$-quadratic modules over $\mathcal{F}$ belonging to $\mathcal{C}$. Then $\Gamma_{\lambda}(\mathcal{F})_{\mathcal{C}}$ is an abelian group under the addition induced by orthogonal sum. Clearly, there is a natural homomorphism

$$
\rho: \Gamma_{\lambda}(\mathcal{F})_{\mathcal{N}} \rightarrow W_{0}^{\lambda}(\underline{A}, \underline{\Lambda}) ;[\kappa: H \rightarrow \underline{H}, \varphi, \mu, \underline{\varphi}] \mapsto[\underline{H}, \underline{\varphi}, \underline{\mu}]
$$

where $W_{0}^{\lambda}(\underline{A}, \underline{\Lambda})$ is the Bak group defined in [5, Definition 1.1]. If $\lambda=(-1)^{k}$ and the $\lambda$-form parameters $\Lambda$ and $\underline{\Lambda}$ are the minimal form parameters of $A$ and $\underline{A}$, respectively, then $\Gamma_{\lambda}(\mathcal{F})_{\mathcal{N}}$ coincides with Cappell-Shaneson's group $\Gamma_{2 k}^{h}(\mathcal{F})$ given in [2, Chapter I, $\S 1]$. 
Theorem 3.1. The natural homomorphism $\iota: \Gamma_{\lambda}(\mathcal{F})_{\mathcal{N}} \rightarrow \Gamma_{\lambda}(\mathcal{F})_{\mathcal{M}}$ is an isomorphism.

Proof. First, we show the injectivity of $\iota$. Let $\alpha$ be an object of $\mathcal{N}$ represents 0 in $\Gamma_{\lambda}(\mathcal{F})_{\mathcal{M}}$. By Lemma 3.3, there exists a $\lambda$-hyperbolic module $\boldsymbol{H}\left(A^{m}\right)$ such that $\alpha \perp \boldsymbol{H}\left(A^{m}\right)$ is a null module. This implies that $\alpha$ represents 0 in $\Gamma_{\lambda}(\mathcal{F})_{\mathcal{N}}$

Next, we prove the surjectivity of $\iota$. Let $\alpha=(\kappa: H \rightarrow \underline{H}, \varphi, \mu, \underline{\varphi})$ be an object in $\mathcal{M}$. Without loss of generality, we can suppose that $\underline{H}$ is a free $\underline{A}$-module. Let $\gamma$ denote the canonical map $H \rightarrow H_{\underline{A}}:=\underline{A} \otimes_{A} H$ and $\alpha_{\underline{A}}$ the induced $\lambda$-quadratic module $\left(\gamma: H \rightarrow H_{\underline{A}}, \varphi, \mu, \varphi_{\underline{A}}\right)$ over $\mathcal{F}$. Since $\underline{H}$ is a free $\underline{A}$-module, take a basis $\left\{y_{1}, \ldots, y_{m}\right\}$ of $\underline{H}$. By multiplying a unit of $\underline{A}$ to $y_{i}$ if necessary, we may assume that all $y_{i}$ lie in the image of $\kappa$. Thus, we can take $x_{1}, \ldots, x_{m}$ in $H$ such that $\kappa\left(x_{1}\right)=y_{1}, \ldots, \kappa\left(x_{m}\right)=y_{m}$. Let $H^{\prime}$ be the free $A$-module with basis $\left\{x_{1}, \ldots, x_{m}\right\}$ and let $\omega: H^{\prime} \rightarrow H$ be the canonical homomorphism. Define $\underline{H}^{\prime}$ to be the $\underline{A}$-submodule of $H_{\underline{A}}$ generated by $\gamma\left(x_{1}\right), \ldots, \gamma\left(x_{m}\right)$. Since $\underline{H}^{\prime} \cong \underline{H}, H_{\underline{A}}^{\prime}$ is naturally isomorphic to $\underline{H}^{\prime}$. Set

$$
\alpha^{\prime}=\left(\gamma_{H^{\prime}}: H^{\prime} \rightarrow \underline{H}^{\prime},\left.\varphi\right|_{H^{\prime} \times H^{\prime}} \circ(\omega \times \omega),\left.\mu\right|_{H^{\prime}} \circ \omega,\left.\varphi_{\underline{A}}\right|_{\underline{H}^{\prime}}\right) .
$$

Then $\alpha^{\prime}$ is an object in $\mathcal{N}$. Moreover, the $A$-submodule $L=\{(\omega(x), x) \in$ $\left.H \oplus H^{\prime} \mid x \in H^{\prime}\right\}$ of $H \oplus H^{\prime}$ is a pre-Lagrangian of $\alpha \perp-\alpha^{\prime}$, and hence $\alpha \sim \alpha^{\prime}$.

From now on, we identify $\Gamma_{\lambda}(\mathcal{F})_{\mathcal{N}}$ and $\Gamma_{\lambda}(\mathcal{F})_{\mathcal{M}}$ with each other and denote them by $\Gamma_{\lambda}(\mathcal{F})$. If $\alpha=(\kappa: H \rightarrow \underline{H}, \varphi, \mu, \underline{\varphi})$ belongs to $\mathcal{N}$, then the datum is abbreviated to $\alpha=(H, \varphi, \mu)$.

Proposition 3.1. Let $\mathcal{F}:(A,-, \lambda, \Lambda) \rightarrow(\underline{A},-, \lambda, \underline{\Lambda})$ and $\mathcal{F}^{\prime}:\left(A^{\prime},-, \lambda\right.$, $\left.\Lambda^{\prime}\right) \rightarrow(\underline{A},-, \lambda, \underline{\Lambda})$ be locally epic homomorphisms. If $\psi: A \rightarrow A^{\prime}$ is a locally epic (resp. strongly locally epic) monomorphism of rings preserving the involution, $\psi(\Lambda)=\psi(A) \cap \Lambda^{\prime}$, and $\mathcal{F}=\mathcal{F}^{\prime} \circ \psi$, then the canonical homomorphism $\psi_{*}: \Gamma_{\lambda}(\mathcal{F}) \rightarrow \Gamma_{\lambda}\left(\mathcal{F}^{\prime}\right)$ is a monomorphism (resp. an isomorphism).

Proof. We regard $A$ as a subring of $A^{\prime}$ via $\psi$.

First, we prove the injectivity of $\psi_{*}$. It suffices to show $\alpha \sim 0$ for an arbitrary $\lambda$-quadratic module $\alpha=(H, \varphi, \mu) \in \mathcal{N}$ over $\mathcal{F}$ such that $\alpha_{A^{\prime}} \sim 0$, where $\alpha_{A^{\prime}}=\left(H_{A^{\prime}}, \varphi_{A^{\prime}}, \mu_{A^{\prime}}\right)$ is the $\lambda$-quadratic module over $\mathcal{F}^{\prime}$ induced by $\psi$ from $\alpha$. By Lemma 3.2, we may suppose that $H$ is a free module over $A$.

By Lemma 3.3, there exists a $\lambda$-hyperbolic module $\boldsymbol{H}\left(A^{\prime s}\right)$ such that $\alpha_{A^{\prime}} \perp \boldsymbol{H}\left(A^{\prime s}\right)$ is a null module. Hence $\alpha_{A^{\prime}} \perp \boldsymbol{H}\left(A^{\prime s}\right)$ has a pre-Lagrangian 
$L^{\prime}$. Suppose $\left\{y_{1}, \ldots, y_{m}\right\}$ generates $L^{\prime}$ over $A^{\prime}$. We regard $A^{s} \subseteq A^{\prime s}$ and $H \subseteq H_{A^{\prime}}$. Since $\psi$ is locally epic, there exists an unit $u$ of $A^{\prime}$ such that $u y_{1}, \ldots, u y_{m} \in H \oplus A^{s} \oplus A^{s}$. Let $L$ denote the $A$-submodule of $H \oplus A^{s} \oplus A^{s}$ generated by $\left\{u y_{1}, \ldots, u y_{m}\right\}$. Since $\Lambda=\Lambda^{\prime} \cap A, L$ is a pre-Lagrangian of $\alpha \perp \boldsymbol{H}\left(A^{s}\right)$, hence we conclude $\alpha \sim 0$.

Next, we prove the surjectivity of $\psi_{*}$ in the strongly locally epic case. Let $\beta=(K, \gamma, \omega)$ be a $\lambda$-quadratic module over $\mathcal{F}^{\prime}$ in $\mathcal{N}_{A^{\prime}}$ such that $K$ is a free $A^{\prime}$-module. Let $\left\{y_{1}, \ldots, y_{m}\right\}$ be an $A^{\prime}$-basis of $K$. Consider the elements $\gamma\left(y_{i}, y_{j}\right)$ and $\widetilde{\omega\left(y_{i}\right)}$ in $A^{\prime}$, where $1 \leq i, j \leq m$ and $\widetilde{\omega\left(y_{i}\right)}$ are liftings of $\omega\left(y_{i}\right)$, respectively. Then there exists an element $u \in A$ such that $u$ is invertible in $A^{\prime}$ and all $u \gamma\left(y_{i}, y_{j}\right), \widetilde{u\left(y_{i}\right)}$ belong to $A$. Set $x_{1}=u y_{1}, \ldots, x_{m}=u y_{m}$, and let $H$ be the $A$-submodule of $K$ generated by $x_{1}, \ldots, x_{m}$. A $\lambda$-Hermitian map $\varphi: H \times H \rightarrow A$ and a $\lambda$-quadratic map $\mu: H \rightarrow A / \Lambda$ are obtained by restricting $\gamma$ and $\omega$ to $H \times H$ and $H$, respectively. Set $\alpha=(H, \varphi, \mu)$. Then $\psi_{*}([\alpha])$ is equal to $[\beta]$.

For a group $G$ and a subset $S$ of $G$, we define

$$
\begin{aligned}
& S(2)=\left\{g \in S \mid g^{2}=e, g \neq e\right\}, \\
& S_{q}=\left\{g \in S \mid g^{2}=e, w(g)=-\lambda\right\}, \\
& S_{s}=S \backslash S_{q} .
\end{aligned}
$$

Theorem 3.2. Let $\widehat{G}=G \ltimes \pi$ be a finite group, $\zeta: \widehat{G} \rightarrow G$ the canonical projection, $\widehat{w}: \widehat{G} \rightarrow\{1,-1\}$ and $w: G \rightarrow\{1,-1\}$ orientation homomorphisms with $\widehat{w}=w \circ \zeta, \lambda=1$ or -1 , and $R=\mathbb{Z}_{(p)}$ for a prime $p$. Let $Q$ be a conjugation-invariant subset of $G(2)$ such that $w(g)=-\lambda$ for all $g \in Q$ and set $\widehat{Q}=\widehat{G}(2) \cap \zeta^{-1}(Q)$. If the order of $\pi$ is prime to $p$, then the canonical homomorphism $\rho: \Gamma_{\lambda}(\mathcal{F}) \rightarrow W_{0}^{\lambda}\left(R[G],(Q)_{R}\right)($ cf. [5, Definition 1.1]) is an isomorphism, where $\mathcal{F}$ is the canonical homomorphism

$$
\left(R[\widehat{G}],-\widehat{w}, \lambda,(\widehat{Q})_{R}\right) \rightarrow\left(R[G],-{ }_{w}, \lambda,(Q)_{R}\right) .
$$

Proof. First, we prove the surjectivity of $\rho$. So, let $\underline{\sigma}$ be an arbitrary element of the group $W_{0}^{\lambda}\left(R[G],(Q)_{R}\right)$ and $\beta=(\underline{H}, \underline{\varphi}, \underline{\mu})$ a $\lambda$-quadratic module representing $\underline{\sigma}$. We may assume that $\underline{H}$ is a free $R[G]$-module and let $\left\{y_{1}, \ldots, y_{m}\right\}$ be an $R[G]$-basis of $\underline{H}$. Then

$$
(\underline{H}, \underline{\varphi}, \underline{\mu}) \perp-(\underline{H}, \underline{\varphi}, \underline{\mu}) \cong \boldsymbol{H}\left(R[G]^{m}\right) .
$$

Let $\left(R[\widehat{G}]^{2 m}, \Phi, M\right)$ stand for the $\lambda$-hyperbolic module $\boldsymbol{H}\left(R[\widehat{G}]^{m}\right)$ and let

$$
\omega: R[\widehat{G}]^{2 m} \rightarrow R[G]^{2 m}
$$


denote the canonical projection. Let $x_{1}, \ldots, x_{m} \in R[\widehat{G}]^{2 m}$ be liftings of $y_{1}, \ldots$, $y_{m}$, respectively. Let $H$ be the free $R[\widehat{G}]$-module with basis $\left\{x_{1}, \ldots, x_{m}\right\}$ and $h: H \rightarrow R[\widehat{G}]^{2 m}$ the canonical homomorphism. Define $\kappa: H \rightarrow \underline{H}, \varphi:$ $H \times H \rightarrow R[\widehat{G}]$, and $\mu: H \rightarrow R[\widehat{G}] /(\widehat{Q})_{R}$ by $\kappa=\omega \circ h, \varphi=\Phi \circ(h \times h)$, and $\mu=M \circ h$, respectively. Then we get the element $\sigma \in \Gamma_{\lambda}(\mathcal{F})$ represented by $\alpha=(\kappa: H \rightarrow \underline{H}, \varphi, \mu, \underline{\varphi})$. Clearly, we have $\rho(\sigma)=\underline{\sigma}$.

Next, we prove the injectivity of $\rho$. Let $\sigma \in \Gamma_{\lambda}(\mathcal{F})$ be an element such that $\rho(\sigma)=0$. We are going to show $\sigma=0$. Let $\alpha=(\kappa: H \rightarrow \underline{H}, \varphi, \mu, \underline{\varphi})$ be a $\lambda$-quadratic module over $\mathcal{F}$ representing $\sigma$. Without loss of generality, we can suppose that $H=R[\widehat{G}]^{m}, \underline{H}=R[G]^{m}$, and $\kappa$ is the canonical homomorphism. Let $\left\{x_{1}, \ldots, x_{m}\right\}$ and $\left\{y_{1}, \ldots, y_{m}\right\}$ be the canonical bases of $H$ and $\underline{H}$, respectively. Define an $R[G]$-homomorphism $\tau: \underline{H} \rightarrow H$ by

$$
\tau\left(y_{i}\right)=\frac{\Sigma_{\pi}}{|\pi|} x_{i}
$$

where

$$
\Sigma_{\pi}=\sum_{c \in \pi} c
$$

Note the property

$$
\tau(\underline{H}) \subseteq H^{\pi}
$$

We shall prove in Step 1 that $\underline{\varphi}\left(y, y^{\prime}\right)=0$ implies $\varphi\left(\tau(y), \tau\left(y^{\prime}\right)\right)=0$, and in Step 2 that $\underline{\varphi}(y, y)=0$ and $\underline{\mu}(y)=0$ imply $\mu(\tau(y))=0$, where $y, y^{\prime} \in \underline{H}$. Once these were shown, we can conclude that if $\alpha_{R[G]}=(\underline{H}, \underline{\varphi}, \underline{\mu})$ has a Lagrangian $L$, then $\tau(L)$ is a pre-Lagrangian of $\alpha$ : namely, $\alpha_{R[G]} \sim 0$ implies $\alpha \sim 0$.

Step 1. Let $\varphi_{e}: H \times H \rightarrow R$ be the $e$-component of $\varphi: H \times H \rightarrow R[\widehat{G}]$ : namely,

$$
\varphi\left(x, x^{\prime}\right)=\sum_{a \in \widehat{G}} \varphi_{e}\left(x, a^{-1} x^{\prime}\right) a
$$

for $x, x^{\prime} \in H$, where $e$ is the identity element of $\widehat{G}$. Let $\zeta_{*}: R[\widehat{G}] \rightarrow R[G]$ denote the homomorphism induced from $\zeta: \widehat{G} \rightarrow G$. By definition, we have

$$
\begin{aligned}
\varphi\left(\tau(y), \tau\left(y^{\prime}\right)\right) & =\sum_{a \in \widehat{G}} \varphi_{e}\left(\tau(y), a^{-1} \tau\left(y^{\prime}\right)\right) a \\
& =\sum_{g \in G}\left(\sum_{a \in \zeta^{-1}(g)} \varphi_{e}\left(\tau(y), a^{-1} \tau\left(y^{\prime}\right)\right) a\right) \\
& =\sum_{g \in G} \varphi_{e}\left(\tau(y), g^{-1} \tau\left(y^{\prime}\right)\right) \Sigma_{\pi} g \quad(\text { by }(3.1)),
\end{aligned}
$$


and

$$
\begin{aligned}
\underline{\varphi}\left(y, y^{\prime}\right) & =\zeta_{*}\left(\varphi\left(\tau(y), \tau\left(y^{\prime}\right)\right)\right) \\
& =\zeta_{*}\left(\sum_{g \in G} \varphi_{e}\left(\tau(y), g^{-1} \tau\left(y^{\prime}\right)\right) \Sigma_{\pi} g\right) \\
& =|\pi| \sum_{g \in G} \varphi_{e}\left(\tau(y), g^{-1} \tau\left(y^{\prime}\right)\right) g .
\end{aligned}
$$

Hence, the equality

$$
\varphi\left(\tau(y), \tau\left(y^{\prime}\right)\right)=\frac{\Sigma_{\pi}}{|\pi|} \underline{\varphi}\left(y, y^{\prime}\right)
$$

holds for all $y, y^{\prime} \in \underline{H}$. Clearly, $\varphi\left(\tau(y), \tau\left(y^{\prime}\right)\right)$ vanishes if and only if $\underline{\varphi}\left(y, y^{\prime}\right)$ does.

Step 2. Suppose $\underline{\varphi}(y, y)=0$ and $\underline{\mu}(y)=0$. By Step 1, we get $\varphi(\tau(y), \tau(y))$ $=0$. Now we decompose $G$ to a disjoint union of the form

$$
G=\{e\} \amalg G(2)_{q} \amalg G(2)_{s} \amalg C \amalg C^{-1}
$$

such that $C^{-1}=\left\{g^{-1} \mid g \in C\right\}$. Set $\mathcal{Q}=\{e\} \cup(G(2) \backslash Q) \cup C$. Define the module $R_{g}, g \in G$, by

$$
R_{g}= \begin{cases}R & \left(g \in G_{s}\right), \\ R / 2 R & \left(g \in G_{q} \backslash Q\right) .\end{cases}
$$

Then we can regard $\underline{\mu}(y)$ as the formal sum

$$
\underline{\mu}(y)=\sum_{g \in \mathcal{Q}} \underline{\mu}_{g}(y) g
$$

with $\underline{\mu}_{g}(y) \in R_{g}$. Similarly choosing a subset $\widehat{\mathcal{Q}}$ of $\widehat{G} \backslash \widehat{Q}$ so that $\zeta(\widehat{\mathcal{Q}})=\mathcal{Q}$, we can write $\mu(x)$ in the form

$$
\mu(x)=\sum_{a \in \widehat{\mathcal{Q}}} \mu_{a}(x) a
$$

with $\mu_{a}(x) \in R_{a}$.

If $a \in \widehat{G}_{s} \cap \widehat{\mathcal{Q}}$, then $\mu_{a}(\tau(y))=0$ follows from $\varphi_{e}\left(\tau(y), a^{-1} \tau(y)\right)=0$.

Next, note that if $p$ is an odd integer and $a \in \widehat{G}_{q} \cap \widehat{\mathcal{Q}}$, then $R_{a}=R / 2 R=0$, and hence $\mu_{a}(\tau(y))=0$. 
It remains to consider $\mu_{a}(\tau(y))$ for $a \in \widehat{G}_{q} \cap \widehat{\mathcal{Q}}$ and $p=2$. In this case, $\pi$ is of odd order. Let $g \in G_{q}$. By definition, we have

$$
\begin{aligned}
\underline{\mu}_{g}(y) & =\sum_{b \in \zeta^{-1}(g) \cap \widetilde{\mathcal{Q}}} \mu_{b}(\tau(y)) \quad \text { in } R / 2 R \\
& =\sum_{b \in \zeta^{-1}(g) \cap \widehat{G}_{q}} \mu_{b}(\tau(y)) \quad(\text { because } \varphi(\tau(y), \tau(y))=0)
\end{aligned}
$$

In particular, if the identity element $e$ belongs to $G_{q}$, then

$$
\underline{\mu}_{e}(y)=\mu_{e}(\tau(y))
$$

since $\pi$ is of odd order. This implies $\mu_{e}(\tau(y))=0$. Suppose $g \in G(2)_{q}$. For $b \in \zeta^{-1}(g) \cap \widehat{G}(2)_{q}$, we can write $b$ in the form $b=c g$ with $c \in \pi$. Note that $g^{2}=e, g \neq e$ and $g c g=c^{-1}$. If $c \neq e$, then the subgroup $\langle c, g\rangle$ generated by $c$ and $g$ is a dihedral group. Since $c\left(c^{u} g\right) c^{-1}=c^{u+2} g$ and

$$
\mu(\tau(y))=\mu(c \tau(y))=c \mu(\tau(y)) \bar{c} \quad(\mathrm{cf.}(3.1)),
$$

we obtain $\mu_{c^{u+2} g}(\tau(y))=\mu_{c^{u} g}(\tau(y))$ and hence $\mu_{b}(\tau(y))=\mu_{g}(\tau(y))$ for all $b \in \zeta^{-1}(g) \cap \widehat{G}(2)_{q}$. Noting that $\left|\zeta^{-1}(g) \cap \widehat{G}_{q}\right|$ is an odd integer, we get

$$
\underline{\mu}_{g}(y)=\mu_{g}(\tau(y))
$$

Thus, we have $\mu_{g}(\tau(y))=0$ as well as $\mu_{b}(\tau(y))=0$ for $b \in \zeta^{-1}(g) \cap \widehat{G}(2)_{q}$.

Putting all together, we have shown $\mu(\tau(y))=0$.

\section{$\S 4$. The $G$-surgery Obstruction and $G$-framed Cobordism Invariance}

Let $X$ be a compact, connected, oriented, smooth $G$-manifold of dimension $n=2 k \geq 6$ satisfying the gap condition (GC) and let $\widetilde{X}$ denote the universal covering space of $X$. The group rings $R[G]$ and $R[\widetilde{G}]$ over a commutative ring $R$ have the involutions derived from the orientation homomorphisms of $X$ and $\widetilde{X}$, respectively (see Section 2). Let $\lambda=(-1)^{k}$. The sets

$$
Q=Q_{X}\left(=\left\{g \in G(2) \mid \operatorname{dim} X^{g}=k-1\right\}\right)
$$

and

$$
\widetilde{Q}=Q_{\widetilde{X}}\left(=\left\{a \in \widetilde{G}(2) \mid \operatorname{dim} \widetilde{X}^{a}=k-1\right\}\right)
$$


generate the $\lambda$-form parameters $(Q)_{R}$ and $(\widetilde{Q})_{R}$ over $R$, respectively. For $\mathbb{Z} \subseteq$ $R \subseteq R^{\prime} \subseteq \mathbb{Q}$, let

$$
\begin{aligned}
& \mathcal{F}_{R, R^{\prime}}:\left(R[\widetilde{G}],-,(-1)^{k},(\widetilde{Q})_{R}\right) \rightarrow\left(R^{\prime}[G],-,(-1)^{k},(Q)_{R^{\prime}}\right), \\
& \mathcal{F}_{R^{\prime}, R^{\prime}}:\left(R^{\prime}[\widetilde{G}],-,(-1)^{k},(\widetilde{Q})_{R^{\prime}}\right) \rightarrow\left(R^{\prime}[G],-,(-1)^{k},(Q)_{R^{\prime}}\right)
\end{aligned}
$$

and

$$
\psi_{R, R^{\prime}}:\left(R[\widetilde{G}],-,(-1)^{k},(\widetilde{Q})_{R}\right) \rightarrow\left(R^{\prime}[\widetilde{G}],-,(-1)^{k},(\widetilde{Q})_{R^{\prime}}\right)
$$

denote the canonical homomorphisms. Then by Proposition 3.1,

$$
\psi_{R, R^{\prime}{ }_{*}}: \Gamma_{\lambda}\left(\mathcal{F}_{R, R^{\prime}}\right) \rightarrow \Gamma_{\lambda}\left(\mathcal{F}_{R^{\prime}, R^{\prime}}\right)
$$

is an isomorphism.

From now on, let $R=\mathbb{Z}$ or $\mathbb{Z}_{(p)}$ for a prime $p$. Let $\boldsymbol{f}=(f, b)$, where $f:(X, \partial X) \rightarrow(Y, \partial Y)$ and $b: T(X) \oplus f^{*} \eta \rightarrow f^{*} \xi$, be an $R$-suitable $G$ framed map (cf. Section 2). Then $\alpha(\boldsymbol{f})=\left(\kappa: \Pi(f) \rightarrow K_{k}(X ; R), \varphi, \mu, \underline{\varphi}\right)$ (see Definition 2.1) is a $\lambda$-quadratic module over $\mathcal{F}_{\mathbb{Z}, R}$. Here we would like to remind readers that $\mathcal{F}_{\mathbb{Z}, R}$ coincides with $\mathcal{F}_{\widetilde{X}, R}$ in Section 1 .

Definition 4.1. We define the element $\sigma(\boldsymbol{f}) \in \Gamma_{\lambda}\left(\mathcal{F}_{\widetilde{X}, R}\right)$ to be the equivalence class of $\alpha(\boldsymbol{f})$.

The element $\sigma(\boldsymbol{f})$ is a $G$-surgery obstruction in the following sense.

Theorem 4.1. Let $R$ and $f$ be as above. If $f$ is $\ell$-connected for some integer such that $1 \leq \ell \leq k-1$ and $\sigma(f)=0$ then one can perform $G$-surgery on $X_{\text {reg }}$ of dimension $k-1$ and $k$ so that the resulting $G$-framed map $f^{\prime}=\left(f^{\prime}, b^{\prime}\right)$ is a degree-one, $\ell$-connected, $R$-homology equivalence, where $f^{\prime}:\left(X^{\prime}, \partial X^{\prime}\right) \rightarrow$ $(Y, \partial Y)$ and $b^{\prime}: T\left(X^{\prime}\right) \oplus f^{\prime *} \eta \rightarrow f^{\prime *} \xi$.

Proof. By hypothesis, $\alpha(\boldsymbol{f}) \sim 0$. By Lemma 3.3, $\alpha(\boldsymbol{f}) \perp \boldsymbol{H}\left(A^{s}\right)$ is a null module for some integer $s \geq 0$. By $s$-iteration of 'trivial' $(k-1)$-dimensional $G$-surgery on $X_{\text {reg }}$ for $\boldsymbol{f}$, we obtain a degree-one, $G$-framed map $\boldsymbol{f}^{\prime \prime}=\left(f^{\prime \prime}, b^{\prime \prime}\right)$ such that $\alpha\left(\boldsymbol{f}^{\prime \prime}\right) \cong \alpha(\boldsymbol{f}) \perp \boldsymbol{H}\left(A^{s}\right)$, where $f^{\prime \prime}:\left(X^{\prime \prime}, \partial X^{\prime \prime}\right) \rightarrow(Y, \partial Y)$ and $b^{\prime \prime}: T\left(X^{\prime \prime}\right) \oplus{f^{\prime \prime *}}^{*} \eta f^{\prime \prime *} \xi$. The resulting map $f^{\prime \prime}: X^{\prime \prime} \rightarrow Y$ is automatically $\ell$-connected. Then by Lemma 2.1 , we can perform $(k-1)$ - and $k$-dimensional $G$-surgery of $f^{\prime \prime}$ on $X_{\text {reg }}^{\prime \prime}$ so that the resulting $f^{\prime}=\left(f^{\prime}, b^{\prime}\right)$ is a degree-one, $\ell$-connected, $R$-homology equivalence, where $f^{\prime}:\left(X^{\prime}, \partial X^{\prime}\right) \rightarrow(Y, \partial Y)$ and $b^{\prime}: T\left(X^{\prime}\right) \oplus f^{\prime *} \eta \rightarrow f^{\prime *} \xi$. 
Let $\boldsymbol{f}$ be as above and $\zeta: \widetilde{G} \rightarrow \widehat{G}$ the projection map, where $\widetilde{G}=\pi_{1}\left(E G \times_{G}\right.$ $X)$ and $\widehat{G}=\pi_{1}\left(E G \times_{G} Y\right)$. Set

$$
\Pi(f)_{\mathbb{Z}[\widehat{G}]}=\mathbb{Z}[\widehat{G}] \otimes_{\mathbb{Z}[\widetilde{G}]} \Pi(f) \text { and } \widehat{Q}=\zeta(\widetilde{Q}) .
$$

Then the canonically induced homomorphism

$$
\widehat{\mathcal{F}}:\left(\mathbb{Z}[\widehat{G}],-, \lambda,(\widehat{Q})_{\mathbb{Z}}\right) \rightarrow\left(R[G],-, \lambda,(Q)_{R}\right)
$$

from $\mathcal{F}_{\mathbb{Z}, R}$ coincides with $\mathcal{F}_{f * \widetilde{Y}, R}$ in Section 1. In the remainder of this section, we study the $\lambda$-quadratic module

$$
\alpha(\boldsymbol{f})_{\mathbb{Z}[\widehat{G}]}=\left(\kappa_{\mathbb{Z}[\widehat{G}]}: \Pi(f)_{\mathbb{Z}[\widehat{G}]} \rightarrow K_{k}(X ; R), \varphi_{\mathbb{Z}[\widehat{G}]}, \mu_{\mathbb{Z}[\widehat{G}]}, \underline{\varphi}\right),
$$

over $\widehat{\mathcal{F}}$ and its equivalence class $\sigma(\boldsymbol{f})_{\mathbb{Z}[\widehat{G}]} \in \Gamma_{\lambda}(\widehat{\mathcal{F}})$.

A $G$-framed cobordism relative to the boundary and the singular set between degree-one, $G$-framed maps $\boldsymbol{f}=(f, b)$ and $\boldsymbol{f}^{\prime}=\left(f^{\prime}, b^{\prime}\right)$ is a degree-one, $G$-framed map $\boldsymbol{F}=(F, B)$ consisting of

$$
F:(W, \partial W) \rightarrow(I \times Y, \partial(I \times Y))
$$

and

$$
B: T(W) \oplus F^{*}\left(\varepsilon_{I}(0) \times \eta\right) \rightarrow F^{*}\left(\varepsilon_{I}(\mathbb{R}) \times \xi\right),
$$

where $I=[0,1]$, satisfying the following:

(1) $\partial W$ has a decomposition $X_{-} \cup X_{0} \cup X_{+}$with $X_{-} \cap X_{+}=\varnothing, X_{-} \cap X_{0}=$ $\partial X_{-}, X_{+} \cap X_{0}=\partial X_{+}$and $\left(X_{-} \cap X_{0}\right) \cup\left(X_{+} \cap X_{0}\right)=\partial X_{0}$ such that $X_{-}=X$ by an orientation-reversing $G$-diffeomorphism, $X_{0}=I \times \partial X$ by a $G$-diffeomorphism, and $X_{+}=X^{\prime}$ by an orientation-preserving $G$ diffeomorphism.

(2) $F\left(X_{-}\right) \subseteq\{0\} \times Y, F\left(X_{0}\right) \subseteq(I \times \partial Y), F\left(X_{+}\right) \subseteq\{1\} \times Y,\left.F\right|_{X_{-}}=f$, $\left.F\right|_{X_{0}}=i d_{I} \times \partial f$ and $\left.F\right|_{X_{+}}=f^{\prime}$.

(3) $\left.B\right|_{X_{-}}=i d_{\varepsilon_{X}(\mathbb{R})} \oplus b,\left.B\right|_{X_{0}}=i d_{\varepsilon_{I}(\mathbb{R})} \times\left. b\right|_{\partial X}$ and $\left.B\right|_{X_{+}}=i d_{\varepsilon_{X^{\prime}}(\mathbb{R})} \oplus b^{\prime}$, where the inward (resp. outward) normal bundle of $X_{-}$(resp. $X_{+}$) in $W$ is identified with $\varepsilon_{X}(\mathbb{R})$ (resp. $\varepsilon_{X^{\prime}}(\mathbb{R})$ ).

(4) There exist a $G$-neighborhood $N_{W}$ of $\bigcup_{H \neq\{e\}} W^{H}$ in $W$, a $G$-neighborhood $N_{X}$ of $\bigcup_{H \neq\{e\}} X^{H}$ in $X$, and a $G$-diffeomorphism $N_{W} \rightarrow I \times N_{X}$ such that $\left.F\right|_{N_{W}}=i d_{I} \times\left. f\right|_{N_{X}}$ and $\left.B\right|_{N_{W}}=i d_{\varepsilon_{I}(\mathbb{R})} \times\left. b\right|_{N_{X}}$. 
Theorem 4.2. $\quad$ Let $R=\mathbb{Z}$ or $\mathbb{Z}_{(p)}$ for a prime $p$, and let $\boldsymbol{f}=(f, b)$, $f:(X, \partial X) \rightarrow(Y, \partial Y)$, and $f^{\prime}=\left(f^{\prime}, b^{\prime}\right), f:\left(X^{\prime}, \partial X^{\prime}\right) \rightarrow(Y, \partial Y)$, be $R$ suitable $G$-framed maps of dimension $n=2 k \geq 6$ such that $X$ and $X^{\prime}$ satisfy the gap condition (GC). In the case $R=\mathbb{Z}$, further suppose

(S6) $f^{H}: X^{H} \rightarrow Y^{H}$ is a $\mathbb{Z}$-homology equivalence for any nontrivial hyperelementary subgroup $H$ of $G$,

or

$\left(\mathrm{S} 6^{\prime}\right) \widetilde{K}_{0}(\mathbb{Z}[G])=0$.

If $\boldsymbol{f}$ and $\boldsymbol{f}^{\prime}$ are $G$-framed cobordant relatively to the boundary and the singular set, then

$$
\sigma(\boldsymbol{f})_{\mathbb{Z}[\widehat{G}]}=\sigma\left(\boldsymbol{f}^{\prime}\right)_{\mathbb{Z}[\widehat{G}]} \quad \text { in } \Gamma_{\lambda}(\widehat{\mathcal{F}}) .
$$

Proof. We prove the theorem under the assumption $\partial X \neq \varnothing$. Since any degree-one, $G$-framed map is $G$-framed cobordant relatively to the boundary and the singular set to a degree-one, $k$-connected, $G$-framed map, it suffices to prove the theorem in the case where $\boldsymbol{f}^{\prime}$ is $k$-connected. Let $\boldsymbol{F}=(F, B)$, $F: W \rightarrow I \times Y$, be a $G$-framed cobordism relative to the boundary and the singular set between $f$ and $f^{\prime}$. By performing $G$-surgery on $W_{\text {reg }}$, we may suppose that $F$ is $k$-connected. Then observe the exact sequence

$$
\cdots \longrightarrow K_{k+1}(W, \partial W) \longrightarrow K_{k}(\partial W) \longrightarrow K_{k}(W) \longrightarrow K_{k}(W, \partial W) \longrightarrow 0,
$$

where the coefficient ring is $R$. By performing handle subtraction for suitable $G$-embeddings $G \times\left(D^{k}, S^{k-1}\right) \rightarrow(W, X)$, we can kill the homology kernel $K_{k}(W, \partial W)$. The procedure changes $\alpha(\boldsymbol{f})$ to $\alpha(\boldsymbol{f}) \oplus \boldsymbol{H}\left(\mathbb{Z}[\widetilde{G}]^{s}\right)$, but it does not change the class $\sigma(\boldsymbol{f})$. Consequently we get the exact sequence

$$
0 \longrightarrow K_{k+1}(W, \partial W) \longrightarrow K_{k}(\partial W) \longrightarrow K_{k}(W) \longrightarrow 0 .
$$

It follows that $K_{k+1}(W, \partial W)$ and $K_{k}(W)$ are stably free $R[G]$-free modules (in the case $R=\mathbb{Z}$, use $(\mathrm{S} 6)$ or $\left.\left(\mathrm{S} 6^{\prime}\right)\right)$. Let $\widetilde{W}$ denote the universal covering space of $W$ and $\widetilde{F}: \widetilde{W} \rightarrow I \times \widetilde{Y}$ the map covering $F$. The covering space $\widehat{X}=f^{*} \widetilde{Y}$ is naturally regarded as a subspace of $\partial \widetilde{W}$ and has the form $\widetilde{X} / N$, where $N$ is a normal subgroup of $\widetilde{G}$ with $\widetilde{G} / N=\widehat{G}$. Let $p_{\widetilde{X}, \widehat{X}}: \widetilde{X} \rightarrow \widehat{X}$ denote the projection map. Let $U$ denote the union of the mapping cylinder $M_{p_{\widetilde{X}, \widehat{X}}}$ of $p_{\widetilde{X}, \widehat{X}}$ and $\widetilde{W}$ attached along $\widehat{X}$. Let $\widetilde{f}: \widetilde{X} \rightarrow \widetilde{Y}$ denote the map covering $f$. Let $(I \times \widehat{X}) \cup \widetilde{W}($ resp. $(I \times X) \cup W)$ denote the space obtained from the disjoint 
union $(I \times \widehat{X}) \amalg \widetilde{W}$ (resp. $(I \times X) \amalg W)$ by canonically identifying $\{1\} \times \widehat{X}$ with $\widehat{X}(\subset \partial \widetilde{W})$ (resp. $\{1\} \times X$ with $X(\subset \partial W)$ ). Then the canonical maps

$$
U \rightarrow(I \times \widehat{X}) \cup \widetilde{W} \text { and } U \rightarrow(I \times X) \cup W
$$

are both branched coverings in general. Let $\partial U$ denote the counter image of $\partial((I \times X) \cup W)$ by the map $U \rightarrow(I \times X) \cup W$. Then $U$ and $\partial U$ are $\widetilde{G}$-spaces. Combining the maps $I \times \widetilde{f}: I \times \widetilde{X} \rightarrow \widetilde{Y}(=\{0\} \times \widetilde{Y})$ and $\widetilde{F}$, we obtain a map $\Psi: U \rightarrow I \times \widetilde{Y}$. Then $\pi_{k+1}\left(\left.\Psi\right|_{\partial U}\right)$ and $\pi_{k+2}\left(\Psi,\left.\Psi\right|_{\partial U}\right)$ are $\mathbb{Z}[\widetilde{G}]$-modules. Thus,

$$
\pi_{k+1}^{\prime}\left(\left.\Psi\right|_{\partial U}\right)=\text { Image }\left[\pi_{k+1}\left(\left.\Psi\right|_{\partial U}\right) \rightarrow \pi_{k+1}\left(\left(\left.\Psi\right|_{\partial U}\right) / N\right)\right]
$$

and

$$
\pi_{k+2}^{\prime}\left(\Psi,\left.\Psi\right|_{\partial U}\right)=\operatorname{Image}\left[\pi_{k+2}\left(\Psi,\left.\Psi\right|_{\partial U}\right) \rightarrow \pi_{k+2}\left(\Psi / N,\left(\left.\Psi\right|_{\partial U}\right) / N\right)\right]
$$

are $\mathbb{Z}[\widetilde{G}]$-modules. Since $U / N$ is a smooth manifold with a bundle datum, $\pi_{k+1}\left(\left(\left.\Psi\right|_{\partial U}\right) / N\right)$ has the ordinary intersection form and the self-intersection form. Moreover, $\pi_{k+1}^{\prime}\left(\left.\Psi\right|_{\partial U}\right)$ has the equivariant intersection form

$$
\varphi_{1}: \pi_{k+1}^{\prime}\left(\left.\Psi\right|_{\partial U}\right) \times \pi_{k+1}^{\prime}\left(\left.\Psi\right|_{\partial U}\right) \rightarrow \mathbb{Z}[\widehat{G}]
$$

and the equivariant self-intersection form

$$
\mu_{1}: \pi_{k+1}^{\prime}\left(\left.\Psi\right|_{\partial U}\right) \rightarrow \mathbb{Z}[\widehat{G}] /(\widehat{Q})_{\mathbb{Z}}
$$

Let $\underline{\varphi_{1}}$ denote the equivariant intersection form on $K_{k}(\partial W)$ over $R[G]$. The canonical homomorphism

$$
\kappa_{1}: \pi_{k+1}^{\prime}\left(\left.\Psi\right|_{\partial U}\right) \rightarrow K_{k}(\partial((I \times X) \cup W))
$$

is locally epic. Set $\alpha_{1}=\left(\kappa_{1}, \varphi_{1}, \mu_{1}, \underline{\varphi_{1}}\right)$. Then $-\alpha(\boldsymbol{f})_{\mathbb{Z}[\widehat{G}]} \perp \alpha\left(\boldsymbol{f}^{\prime}\right) \sim \alpha_{1}$. Clearly the image of $\pi_{k+2}^{\prime}\left(\Psi,\left.\Psi\right|_{\partial U}\right)$ in $\overline{\pi_{k+1}^{\prime}}\left(\left.\Psi\right|_{\partial U}\right)$ is a pre-Lagrangian of $\alpha_{1}$. Thus, $\sigma(\boldsymbol{f})_{\mathbb{Z}[\widehat{G}]}=\sigma\left(\boldsymbol{f}^{\prime}\right)_{\mathbb{Z}[\widehat{G}]}$ in $\Gamma_{\lambda}(\widehat{\mathcal{F}})$.

If $\partial X=\varnothing$, we reduce the proof to the case with boundary. Let $y_{0}$ be a point in $Y_{\text {reg. }}$. Then $G$-acts freely on the $G$-orbit $G y_{0}$. Without loss of generality, we can suppose $f$ is transverse regular to $\left\{y_{0}\right\}$. Since $f$ is of degree one, the number of points in $f^{-1}\left(y_{0}\right)$ odd. Since $f: X \rightarrow Y$ is one connected, we can modify $f: X \rightarrow Y$ by $G$-homotopy deformation relative to boundary so that $f^{-1}\left(y_{0}\right)$ consists of exactly one point, $x_{0}$ say. Similarly we may assume that $f^{\prime}$ is transverse regular to $\left\{y_{0}\right\}$ and $f^{\prime-1}\left(y_{0}\right)$ consists of exactly one point, $x_{0}^{\prime}$ say. Next, we may suppose that $F: W \rightarrow I \times Y$ is transverse regular to $I \times\left\{y_{0}\right\}$. Let 
$L$ be the connected component of $F^{-1}\left(I \times\left\{y_{0}\right\}\right)$ containing $x_{0}$ and $x_{0}^{\prime}$. Each connected component of $F^{-1}\left(I \times\left\{y_{0}\right\}\right)$ except $L$ is diffeomorphic to a circle. Thus, we can remove the circle components by one-dimensional $G$-surgery on $W_{\text {reg }}$ of $(F, B)$. Removing appropriate tiny open $G$-tubular neighborhoods $T$ and $T^{\prime}$ of $G \cdot L$ and $G y_{0}$ from $W$ and $Y$, respectively, we obtain $G$-framed cobordism $\boldsymbol{F}_{0}=\left(F_{0}, B_{0}\right)$ consisting of

$$
F_{0}:\left(W_{0}, \partial W_{0}\right) \rightarrow\left(I \times Y_{0}, \partial\left(I \times Y_{0}\right)\right)
$$

and

$$
B_{0}: T\left(W_{0}\right) \oplus F_{0}^{*}\left(\varepsilon_{I}(\mathbb{R}) \times \eta\right) \rightarrow F_{0}^{*}\left(\varepsilon_{I}(\mathbb{R}) \times \xi\right)
$$

between degree-one, $G$-framed maps $\boldsymbol{f}_{0}$ and $\boldsymbol{f}_{0}^{\prime}$ such that $W_{0}=W \backslash T, Y_{0}=Y \backslash$ $T^{\prime}, \sigma\left(\boldsymbol{f}_{0}\right)=\sigma(\boldsymbol{f})$ and $\sigma\left(\boldsymbol{f}_{0}^{\prime}\right)=\sigma\left(\boldsymbol{f}^{\prime}\right)$. Since the relevant underlying manifolds of these $G$-framed maps have boundaries, we get $\sigma\left(\boldsymbol{f}_{0}\right)=\sigma\left(\boldsymbol{f}_{0}^{\prime}\right)$.

\section{$\S 5$. Proofs of Theorems 1.1 and 1.2}

Let $R$ denote either $\mathbb{Z}$ or $\mathbb{Z}_{(p)}$ for a prime $p$. Let $\boldsymbol{f}=(f, b)$ be the $G$ framed map in Theorem 1.1. In the case where $f$ itself is $R$-suitable, we defined the element $\sigma(\boldsymbol{f}) \in \Gamma_{(-1)^{k}}\left(\mathcal{F}_{\mathbb{Z}, R}\right)$ in Definition 4.1. But in general, we can perform $G$-surgery of $\boldsymbol{f}$ on $X_{\text {reg }}$ so that the resulting degree-one, $G$-framed map $f_{k}=\left(f_{k}, b_{k}\right), f_{k}:\left(X_{k}, \partial X_{k}\right) \rightarrow(Y, \partial Y)$, is $k$-connected. Then by definition, $f_{k}$ is $R$-suitable. Thus we obtain the element $\sigma\left(f_{k}\right) \in \Gamma_{(-1)^{k}}\left(\mathcal{F}_{\widetilde{X}_{k}, R}\right)$. Since $\pi_{1}\left(E G \times{ }_{G} X_{k}\right)$ is canonically isomorphic to $\widehat{G}:=\pi_{1}\left(E G \times{ }_{G} Y\right)$, we can identify $\mathcal{F}_{\widetilde{X_{k}}, R}$ with $\mathcal{F}_{f * \widetilde{Y}, R}$ in Section 1 as well as $\widehat{\mathcal{F}}$ in Section 4 .

Definition 5.1. We define $\sigma_{\mathrm{CS}}(\boldsymbol{f}) \in \Gamma_{(-1)^{k}}\left(\mathcal{F}_{f^{*} \widetilde{Y}, R}\right)$ to be the element $\sigma\left(\boldsymbol{f}_{k}\right)$.

By Theorem 4.2, $\sigma_{\mathrm{CS}}(\boldsymbol{f})$ is defined independently of choice of $\boldsymbol{f}_{k}$.

Proof of Theorem 1.1. Let $f=(f, b)$ be the $G$-framed map in Theorem 1.1 and let $f_{k}=\left(f_{k}, b_{k}\right)$ be as above.

(i) Let $\boldsymbol{f}^{\prime}$ be a degree-one, $G$-framed map. As in the case for $\boldsymbol{f}$, we have a degree-one, $k$-connected, $G$-framed map $f_{k}^{\prime}$ which is $G$-framed cobordant relatively to the boundary and the singular set to $f^{\prime}$. Then by definition, $\sigma_{\mathrm{CS}}\left(\boldsymbol{f}^{\prime}\right)=\sigma\left(\boldsymbol{f}_{k}^{\prime}\right)$. If $\boldsymbol{f}$ is $G$-framed cobordant relatively to the boundary and the singular set to $\boldsymbol{f}^{\prime}$ then $\boldsymbol{f}_{k}$ is so to $\boldsymbol{f}_{k}^{\prime}$. By Theorem 4.2, $\sigma\left(\boldsymbol{f}_{k}\right)=\sigma\left(\boldsymbol{f}_{k}^{\prime}\right)$ and hence $\sigma_{\mathrm{CS}}(\boldsymbol{f})=\sigma_{\mathrm{CS}}\left(\boldsymbol{f}^{\prime}\right)$. 
(ii) Assume $f$ is a one-connected, $R$-homology equivalence. Then $f$ is $R$ suitable. We immediately obtain $\sigma(\boldsymbol{f})_{\mathbb{Z}[\widehat{G}]}=0$. By Theorem 4.2, $\sigma(\boldsymbol{f})_{\mathbb{Z}[\widehat{G}]}=$ $\sigma\left(\boldsymbol{f}_{k}\right)$. Thus $\sigma_{\mathrm{CS}}(\boldsymbol{f})=\sigma\left(\boldsymbol{f}_{k}\right)=0$.

(iii) Suppose $\sigma_{\mathrm{CS}}(\boldsymbol{f})=0$. The equality $\sigma\left(\boldsymbol{f}_{k}\right)=0$ immediately follows. Thus, by Theorem 4.1 , we can convert $f_{k}$ by $G$-surgery on $X_{k \text { reg }}$ to a $(k-1)$ connected $R$-homology equivalence $f^{\prime}$.

In the following (iv)-(v), we suppose $f$ is $R$-suitable.

(iv) Suppose $\sigma(\boldsymbol{f})=0$ and $f$ is $\ell$-connected. By Theorem 4.1, we can convert $f$ by $(k-1)$ - and $k$-dimensional $G$-surgery on $X_{\text {reg }}$ to an $\ell$-connected $R$-homology equivalence $f^{\prime}$.

(v) By Theorem 4.2, $\sigma\left(\boldsymbol{f}_{k}\right)=\sigma(\boldsymbol{f})_{\mathbb{Z}[\widehat{G}]}$. Thus we obtain $\sigma_{\mathrm{CS}}(\boldsymbol{f})=\sigma\left(\boldsymbol{f}_{k}\right)=$ $\sigma(\boldsymbol{f})_{\mathbb{Z}[\widehat{G}]}$.

Proof of Theorem 1.2. Let $f$ and $f^{\prime}$ be as in Theorem 1.2. By Theorem 4.2 , we can assume without loss of generality that $\boldsymbol{f}$ and $\boldsymbol{f}^{\prime}$ are $k$-connected. Set

$$
\begin{aligned}
& X^{\prime \prime}=X \#_{G, x_{1}} G \times_{H} \Delta X^{\prime}, \\
& f^{\prime \prime}=f \#_{G, x_{1}} G \times_{H} \Delta f^{\prime}, \\
& b^{\prime \prime}=b \#_{G, x_{1}} G \times_{H} \Delta b^{\prime}, \\
& f^{\prime \prime}=f \#_{G, x_{1}} G \times_{H} \Delta f^{\prime} .
\end{aligned}
$$

Recall $\Delta X^{\prime}=(-Y) \cup_{\partial(-Y)} X^{\prime}$. Thus by assumption, $\pi_{1}\left(X^{\prime}\right)=\pi_{1}\left(\Delta X^{\prime}\right)=$ $\pi_{1}(Y)$. If we forget the $G$-actions, $X^{\prime \prime}$ is the ordinary connected sum of manifolds $X$ and $|G / H|$ copies of $\Delta X^{\prime}$. Clearly, $f^{\prime \prime}$ is one-connected and

$$
K_{k}\left(X^{\prime \prime} ; R\right)=K_{k}(X ; R) \oplus \operatorname{Ind}_{H}^{G} K_{k}\left(\operatorname{Res}_{H}^{G} X^{\prime} ; R\right) .
$$

According to the definition of the $G$-surgery obstruction, we have the $(-1)^{k}$ quadratic modules

$$
\alpha(\boldsymbol{f})=\left(\kappa: \Pi(f) \rightarrow K_{k}(X ; R), \varphi, \mu, \underline{\varphi}\right)
$$

over $\mathcal{F}_{\widetilde{X}, R}\left(=\mathcal{F}_{f * \widetilde{Y}, R}\right.$ in this situation) associated with $\boldsymbol{f}$,

$$
\alpha\left(f^{\prime}\right)=\left(\kappa^{\prime}: \Pi\left(f^{\prime}\right) \rightarrow K_{k}\left(X^{\prime} ; R\right), \varphi^{\prime}, \mu^{\prime}, \underline{\varphi^{\prime}}\right)
$$

over $\mathcal{F}_{\widetilde{X}^{\prime}, R}\left(=\mathcal{F}_{f^{\prime *} \widetilde{Y}, R}\right.$ in this situation) associated with $f^{\prime}$, and

$$
\alpha\left(f^{\prime \prime}\right)=\left(\kappa^{\prime \prime}: \Pi\left(f^{\prime \prime}\right) \rightarrow K_{k}\left(X^{\prime \prime} ; R\right), \varphi^{\prime \prime}, \mu^{\prime \prime}, \underline{\varphi^{\prime \prime}}\right)
$$


over $\mathcal{F}_{\widetilde{X^{\prime \prime}}, R}$ associated with $f^{\prime \prime}$. Then $\Pi\left(f^{\prime \prime}\right)_{\mathbb{Z}[\widehat{G}]}$ canonically contains

$$
\Pi(f) \oplus \operatorname{Ind}_{\widehat{H}}^{\widehat{G}} \Pi\left(\operatorname{Res}_{H}^{G} f^{\prime}\right),
$$

where

$$
\operatorname{Ind}_{\widehat{H}}^{\widehat{G}} \Pi\left(\operatorname{Res}_{H}^{G} f^{\prime}\right)=\mathbb{Z}[\widehat{G}] \otimes_{\mathbb{Z}[\widehat{H}]} \Pi\left(\operatorname{Res}_{H}^{G} f^{\prime}\right)
$$

Clearly,

$$
\kappa_{\mathbb{Z}[\widehat{G}]}^{\prime \prime}\left(\Pi(f) \oplus \operatorname{Ind}_{\widehat{H}}^{\widehat{G}} \Pi\left(\operatorname{Res}_{H}^{G} f^{\prime}\right)\right)=K_{k}\left(X^{\prime \prime} ; R\right) .
$$

Let $\boldsymbol{\psi}$ denote the pair $(\psi, \underline{\psi})$ consisting of the canonical homomorphisms

$$
\psi:\left(\mathbb{Z}[\widehat{H}],(-1)^{k},-,\left(Q_{\operatorname{Res} \frac{\widehat{G}}{\widehat{X}^{\prime}}}\right)_{\mathbb{Z}}\right) \rightarrow\left(\mathbb{Z}[\widehat{G}],(-1)^{k},-,\left(Q_{\widetilde{X^{\prime}}}\right)_{\mathbb{Z}}\right)
$$

and

$$
\underline{\psi}:\left(R[H],(-1)^{k},-,\left(Q_{\operatorname{Res}_{H}^{G} X^{\prime}}\right)_{R}\right) \rightarrow\left(R[G],(-1)^{k},-,\left(Q_{X^{\prime}}\right)_{R}\right) .
$$

As in [7], [3] (particularly, (6.3) and the definition of $\varphi_{\#} q$ prior to Lemma 12.3 in [7]), we can define the $(-1)^{k}$-quadratic module $\boldsymbol{\psi}_{\#} \alpha\left(\operatorname{Res}_{H}^{G} \boldsymbol{f}^{\prime}\right)$ over $\mathcal{F}_{f^{\prime *} \widetilde{Y}, R}$, and denote it by $\operatorname{Ind}_{(\widehat{H}, H)}^{(\widehat{G}, G)} \alpha\left(\operatorname{Res}_{H}^{G} \boldsymbol{f}^{\prime}\right)$. It follows from Lemma 3.1 that $\alpha\left(\boldsymbol{f}^{\prime \prime}\right)_{\mathbb{Z}[\widehat{G}]}$ is equivalent to

$$
\alpha(\boldsymbol{f}) \perp \operatorname{Ind}_{(\widehat{H}, H)}^{(\widehat{G}, G)} \alpha\left(\operatorname{Res}_{H}^{G} \boldsymbol{f}^{\prime}\right) .
$$

Thus we conclude $\sigma_{\mathrm{CS}}\left(\boldsymbol{f}^{\prime \prime}\right)=\sigma_{\mathrm{CS}}(\boldsymbol{f})+\operatorname{Ind}_{(\widehat{H}, H)}^{(\widehat{G}, G)} \sigma_{\mathrm{CS}}\left(\operatorname{Res}_{H}^{G} \boldsymbol{f}^{\prime}\right)$.

\section{References}

[1] Bak, A., K-theory of Forms, Ann. of Math. Stud., 98, Princeton Univ. Press, 1981.

[2] Cappell, S. E. and Shaneson, J. L., The codimension two placement problem and homology equivalent manifolds, Ann. of Math., 99 (1974), 277-348.

[3] Ju, X. M., Matsuzaki, K. and Morimoto, M., Mackey and Frobenius structures on odd dimensional surgery obstruction groups, K-Theory, 29 (2003), 285-312.

[4] Morimoto, M. and Iizuka, K., Extendibility of $G$-maps to pseudoequivalences to finite G-CW-complexes whose fundamental groups are finite, Osaka J. Math., 21 (1984), 5969.

[5] Morimoto, M., Bak groups and equivariant surgery, K-Theory, 2 (1989), 465-483.

[6] _ Bak groups and equivariant surgery II, K-Theory, 3 (1990), 505-521.

[7] - Induction theorems of equivariant surgery obstruction groups, Trans. Amer. Math. Soc., 355 (2003), 2341-2384.

[8] - Deleting and inserting fixed point sets on disks under the strong gap condition, K-theory Preprint Archives, no. 707 (September, 2004).

[9] Oliver, B., Fixed point sets and tangent bundles of actions on disks and euclidean spaces, Topology, 35 (1996), 583-615.

[10] Wall, C. T. C., Surgery on Compact Manifolds, Academic Press, London-New York, 1970. 